\title{
Penalty Function-Based Joint Diagonalization Approach for Convolutive Blind Separation of Nonstationary Sources
}

\author{
Wenwu Wang, Member, IEEE, Saeid Sanei, Member, IEEE, and Jonathon A. Chambers, Senior Member, IEEE
}

\begin{abstract}
A new approach for convolutive blind source separation (BSS) by explicitly exploiting the second-order nonstationarity of signals and operating in the frequency domain is proposed. The algorithm accommodates a penalty function within the cross-power spectrum-based cost function and thereby converts the separation problem into a joint diagonalization problem with unconstrained optimization. This leads to a new member of the family of joint diagonalization criteria and a modification of the search direction of the gradient-based descent algorithm. Using this approach, not only can the degenerate solution induced by a null unmixing matrix and the effect of large errors within the elements of covariance matrices at low-frequency bins be automatically removed, but in addition, a unifying view to joint diagonalization with unitary or nonunitary constraint is provided. Numerical experiments are presented to verify the performance of the new method, which show that a suitable penalty function may lead the algorithm to a faster convergence and a better performance for the separation of convolved speech signals, in particular, in terms of shape preservation and amplitude ambiguity reduction, as compared with the conventional second-order based algorithms for convolutive mixtures that exploit signal nonstationarity.
\end{abstract}

Index Terms-Blind source separation, convolutive mixtures, frequency domain, orthogonal/nonorthogonal constraints, penalty function, speech signals.

\section{INTRODUCTION}

$\mathbf{T}$ HE objective of blind source separation (BSS) is to extract the original source signals of interest from their mixtures and possibly to estimate the unknown mixing channel using only the information within the mixtures observed at the output of each channel with no, or very limited, knowledge about the source signals and the mixing channel. A challenging BSS problem is to separate convolutive mixtures of source signals, where the observed signals are assumed to be the mixtures of linear convolutions of unknown sources. This is an issue in several application fields, of which the most famous is the cocktail party problem, where the name comes from the fact that we can hold a conversation at a cocktail party even though other people are speaking at the same time within an enclosed

Manuscript received September 11, 2003; revised May 15, 2004. This work was supported by the Engineering and Physical Sciences Research Council (EPSRC) of the United Kingdom. The associate editor coordinating the review of this manuscript and approving it for publication was Dr. Hamid Krim.

The authors are with the Cardiff School of Engineering, Cardiff University, Cardiff, CF24 0YF, U.K. (e-mail: wenwu.wang@ieee.org; saneis@cf.ac.uk; chambersj@cf.ac.uk).

Digital Object Identifier 10.1109/TSP.2005.845433 environment [1]; this classical definition can be extended to include the presence of a noise background due to the other interfering speech or music. In this case, the observed signals are the mixtures of weighted and delayed components of the sources.

Convolutive BSS has been conventionally developed in either the time domain (see [2] and the reference therein) or the frequency domain, e.g., [3]-[18]. In this paper, we focus on the operation in the frequency domain. In contrast to time domain approaches, where a large number of parameters has to be estimated, frequency domain approaches usually simplify the convolutive problem into an application of instantaneous methods to each frequency bin. As a result, the frequency domain approaches generally have a simpler implementation and better convergence performance, although the downside of arbitrary permutations and scaling ambiguities of the recovered frequency response of the sources at each frequency bin remain open problems in many realistic environments, such as where the sources are moving. The representative separation criterion used in the frequency domain is the cross-power spectrum-based cost function, where $\mathbf{W}(\omega)=0$ (shown in Section II) generally leads to a trivial solution to the minimization of the cost function. A selective method, as has been used in some general ICA approaches, is to incorporate a term of the form $\log \left|\operatorname{det}\left(\mathbf{W}(\omega \mathbf{W})^{T}(\omega)\right)\right|$ in the cost function to be minimized to ensure that the determinant does not approach zero. Alternatively, one can also use a special initialization method or a hard constraint to the parameters to avoid this degenerate solution.

In this paper, in contrast, we propose a new approach based on a penalty function for convolutive blind separation in the frequency domain. This new criterion is motivated by nonlinear programming techniques in optimization. As will be shown, incorporating a penalty function within the second-order statistics (SOS)-based cost function for nonstationary signals, the degenerate solution due to $\mathbf{W}(\omega)$ approaching zero is automatically removed. Another objective of this paper is to use a penalty function to unify the concept of joint diagonalization with unitary or nonunitary constraint. Under such a framework, the constrained joint diagonalization problem is converted into an unconstrained optimization problem, and the main task is then to focus on the choice of the form of penalty functions associated with appropriate application models and the adaptation of the penalty parameters. This is particularly useful for applying some well-known optimization algorithms and therefore developing new algorithms for BSS. Moreover, such a criterion has 
a close relation to the joint diagonalization methods with projection onto the Stefiel manifold. The state-of-the-art schemes, using either orthogonal or nonorthogonal matrix constraints, appear to be related to the new criterion and may lead to new viewpoints and algorithms for joint diagonalization regarding conventional optimization methods such as the Newton and gradient descent algorithms. As will be shown in Sections VI and VII, the penalty function may lead to improved performance for the solution of the BSS problem, and the implementation of a nonunitary constraint in a later example indicates an additional ability of the proposed scheme to reduce the scaling ambiguities.

In this paper, we assume that the source signals are nonstationary. This is especially true for most real-world signals whose statistical properties are very often statistically nonstationary or (quasi-) cyclostationary, such as speech and biological signals. In [26], we have shown that the cyclostationarity of the signals can be exploited in order to generate new algorithms that outperform some conventional BSS algorithms for some real-world cyclic-like signals such as ECGs. However, as observed in [26], cyclostationarity may not be valid for speech signals whose statistics vary very rapidly with time. In this case, it is difficult to estimate accurately their cycle frequencies. A natural and better way to describe speech signals is to consider their nonstationarity. To this end, we exploit the SOS of the covariance matrices to estimate the separation matrix. In comparison to higher order statistic (HOS) methods, although using SOS is insufficient for separation of stationary signals [19], and therefore, HOS has to be considered either explicitly [20] or implicitly [3], SOS methods usually have a simpler implementation and better convergence performance and require fewer data samples for time-averaged estimates of the SOSs. Moreover, they can potentially overcome the non-Gaussianity assumption for source signals [5], which is nevertheless necessary for HOS-based methods.

This paper is organized as follows. In Section II, the convolutive BSS problem and the frequency domain approach are discussed. A brief discussion on the problem of second-order nonstationarity of speech signals and the off-diagonal separation criterion is given in Section III. In Section IV, several joint diagonalization criteria are summarized, and the concept of constrained BSS is introduced. This provides the fundamental background for this work. In Section V, the penalty function-based joint diagonalization method is described in detail. In Section VI, a unifying analysis will be given to the unitary and nonunitary constraints within joint diagonalization under the penalty function framework. An implementation example with gradient adaptation is also included in this section. Section VII presents the simulation results, and Section VIII concludes the paper.

\section{Convolutive BSS in the FreQuency Domain}

Assume that $N$ source signals are recorded by $M$ microphones (here, we are particularly interested in acoustic applications), where $M \geq N$. The output of the $j$ th microphone is modeled as a weighted sum of convolutions of the source signals corrupted by additive noise, that is

$$
x_{j}(n)=\sum_{i=1}^{N} \sum_{p=0}^{P-1} h_{j i p} s_{i}(n-p)+v_{j}(n)
$$

where $h_{j i p}$ is the $p$ th element of the $P$-point impulse response from source $i$ to microphone $j(j=1, \ldots, M), s_{i}$ is the signal from source $i, x_{j}$ is the signal received by microphone $j, v_{j}$ is the additive noise, and $n$ is the discrete time index. All signals are assumed zero mean. Throughout the paper, both the mixing and unmixing processes are assumed to be causal finite filter models, i.e., FIR filters as in (1). We note that it is also possible to exploit noncausal models [27], [28], but this issue is, however, beyond the scope of this paper. Using a $T$-point windowed discrete Fourier transformation (DFT), time-domain signals $x_{j}$ can be converted into frequency-domain time-series signals as

$$
X_{j}(\omega, t)=\sum_{\tau=0}^{T-1} x_{j}(t+\tau) w(\tau) e^{-J 2 \pi \omega \tau}
$$

where $w(\tau)$ denotes a window function, $J=\sqrt{-1}$, and $\omega$ is a frequency index $\omega=0,1 / T, \ldots,((T-1) / T)$. We use closed-form expressions for $H_{j i}(\omega), S_{i}(\omega, t)$, and $V_{i}(\omega, t)$, in which $H_{j i}(\omega)$ does not depend on the time index $t$ due to the assumption that the mixing system is time invariant. The same assumption will be applied to the separation system as follows. As shown in [4], a linear convolution can be approximated by a circular convolution if $P \ll T$, that is

$$
\mathbf{X}(\omega, t)=\mathbf{H}(\omega) \mathbf{S}(\omega, t)+\mathbf{V}(\omega, t)
$$

where $\mathbf{S}(\omega, t)=\left[S_{1}(\omega, t), \ldots, S_{N}(\omega, t)\right]^{T}$ and $\mathbf{X}(\omega, t)=$ $\left[X_{1}(\omega, t), \ldots, X_{M}(\omega, t)\right]^{T}$ are the time-frequency representations of the source signals and the observed signals, respectively, and $(\cdot)^{T}$ denotes vector transpose. Equation (3) implies that the problem of convolutive BSS has been transformed into multiple instantaneous (but complex-valued) BSS problems at each frequency bin.

Rather than directly estimating $\mathbf{H}(\omega)$, we can alternatively estimate a weighted pseudo-inverse of $\mathbf{H}(\omega)$, i.e., $\mathbf{W}(\omega)$ at every frequency bin $\omega$ by using a backward discrete-time model [15], [16]

$$
\mathbf{Y}(\omega, k)=\mathbf{W}(\omega) \mathbf{X}(\omega, k)
$$

where $\mathbf{Y}(\omega, k)=\left[Y_{1}(\omega, k), \ldots, Y_{N}(\omega, k)\right]^{T}$ is the time-frequency representation of the estimated source signals, and $k$ is the discrete time index. The parameters in $\mathbf{W}(\omega)$ are determined so that the elements $Y_{1}(\omega, k), \ldots, Y_{N}(\omega, k)$ become mutually independent. The above calculations can be carried out independently in each frequency bin.

\section{SECOND-ORDER NONSTATIONARITY AND OFF-DIAGONAL CRITERION}

Define a cost function $\mathcal{J}(\mathbf{W}(\omega))$ as a measure of independence between $Y_{i}(\omega, k)$, for $i=1, \ldots, N$; the separation problem then becomes an optimization problem for which we have to find a method to minimize $\mathcal{J}(\mathbf{W}(\omega))$ so that $Y_{i}(\omega, k)$ 
are as independent as possible. A necessary condition to exploit the SOS conditions for nonstationary signals is to ensure that the $Y_{i}(\omega, k)$ are mutually uncorrelated. Nonstationarity of speech signals can be generated in various ways, e.g., variation of the vocal tract filter and glottis, whereby neighboring samples have varying cross correlation, or even higher order moments. Here, we resort to the cross-power spectrum of the output signals at multiple times, i.e.,

$$
\mathbf{R}_{Y}(\omega, k)=\mathbf{W}(\omega)\left[\mathbf{R}_{X}(\omega, k)-\mathbf{R}_{V}(\omega, k)\right] \mathbf{W}^{H}(\omega)
$$

where $\mathbf{R}_{X}(\omega, k)$ is the covariance matrix of $\mathbf{X}(\omega, k)$, and $\mathbf{R}_{V}(\omega, k)$ is the covariance matrix of $\mathbf{V}(\omega, k)$. The objective is to find a $\mathbf{W}(\omega)$ that (at least approximately) jointly diagonalizes these matrices simultaneously for all time blocks $k, k=1, \ldots, K$. That is, $\mathbf{R}_{Y}(\omega, k) \rightarrow \Lambda_{C}(\omega, k)$, where $\boldsymbol{\Lambda}_{C}(\omega, k)$ is an arbitrary diagonal matrix, which, based on the independence assumptions of the source signals $s_{i}(i=1, \ldots, N)$ and the sensor noise, can be derived by the following equation:

$$
\mathbf{R}_{X}(\omega, k)-\mathbf{R}_{V}(\omega, k)=\mathbf{H}(\omega) \boldsymbol{\Lambda}_{S}(\omega, k) \mathbf{H}^{H}(\omega)-\boldsymbol{\Lambda}_{V}(\omega, k)
$$

where $\boldsymbol{\Lambda}_{S}(\omega, k)$ and $\boldsymbol{\Lambda}_{V}(\omega, k)$ are the different diagonal covariance matrices of the source and noise signals for each $k$, respectively, and $(\cdot)^{H}$ denotes the Hermitian transpose operator. As in [4], an intuitive criterion is defined to minimize a function of the error $E(\omega, k)$ between $\boldsymbol{\Lambda}_{S}(\omega, k)$ and $\mathbf{R}_{Y}(\omega, k)$

$$
\mathcal{J}(\mathbf{W}(\omega))=\underset{\mathbf{W}}{\operatorname{argmin}} \sum_{\omega=1}^{T} \sum_{k=1}^{K}\|E(\omega, k)\|_{F}^{2}
$$

where $\|\cdot\|_{F}^{2}$ is the squared Frobenius norm. It is straightforward to show that this separation criterion is equivalent to (denoted by problem $P_{1}$ )

$$
P_{1}: \mathcal{J}(\mathbf{W}(\omega))=\underset{\mathbf{W}}{\operatorname{argmin}} \sum_{\omega=1}^{T} \sum_{k=1}^{K} \mathcal{F}(\mathbf{W})(\omega, k)
$$

where $\mathcal{F}(\mathbf{W})(\omega, k)$ is defined as

$$
\mathcal{F}(\mathbf{W})(\omega, k)=\left\|\mathbf{R}_{Y}(\omega, k)-\operatorname{diag}\left[\mathbf{R}_{Y}(\omega, k)\right]\right\|_{F}^{2}
$$

where $\operatorname{diag}(\cdot)$ is an operator that zeros the off-diagonal elements of a matrix.

The solution $\mathbf{W}(\omega)=\mathbf{0}$ clearly leads to the minimization of $\mathcal{F}(\mathbf{W})(\omega, k)$, which is a degenerate solution to the minimization of $\mathcal{J}(\mathbf{W})$. This means that a cost function sufficient for joint diagonalization should enforce a solution for $\mathbf{W}(\omega) \neq \mathbf{0}$ and, hence, a constraint on the former cost function. On the other hand, the representative approaches (mostly working in the time domain) using joint diagonalization project the separation matrix $\mathbf{W}$ onto the Stefiel manifold to generate a unitary constraint (cf. [21], $\mathbf{W} \mathbf{W}^{H}=\mathbf{I}$ ). Nevertheless, as shown in [22], such a unitary constraint is not always necessary $\left(\mathbf{W} \mathbf{W}^{H} \neq \mathbf{I}\right)$. Unlike the method using a hard constraint on the initialization or at each iteration (see [4]), here, we present an alternatively new approach to satisfy the constraint $\mathbf{W}(\omega) \neq \mathbf{0}$. This method is motivated by nonlinear programming optimization theory [23] and, at the same time, unifies the orthogonal and nonorthogonal matrix constraint methods and therefore provides new insight into joint diagonalization algorithms for BSS (see Section VI). For simplicity, the frequency index $\omega$ in $\mathbf{W}(\omega)$ is omitted hereafter where appropriate.

\section{Joint Diagonalization CRITERIA AND CONSTRAINED BSS}

\section{A. Criteria}

The objective of joint (approximate) diagonalization is to find a matrix that simultaneously (approximately) diagonalizes a set of matrices. The typical application of joint diagonalization for BSS is to achieve separation by using (very often approximating) joint diagonalization of a set of covariance matrices. In comparison with the method using only one covariance matrix, approximate joint diagonalization of a set of covariance matrices increases the statistical reliability of the procedure for estimating the unmixing matrix and reduces the possibility of unsuccessful separation induced by choosing an incorrect time-lag of covariance matrices, therefore significantly increasing the robustness at the cost of increased computation. Letting the set of matrices to be jointly diagonalized be $\overline{\mathbf{R}}_{k}(k=1, \ldots, K)$, the widely used off-diagonal criterion for the joint diagonalization stated in Section III can be expressed generally as

$$
\mathcal{J}(\mathbf{W})=\underset{\mathbf{W}}{\operatorname{argmin}} \sum_{k=1}^{K} \operatorname{off}\left(\mathbf{W}^{H} \overline{\mathbf{R}}_{k} \mathbf{W}\right)
$$

where $\mathbf{W}$ is a joint diagonalizer, which is also referred to as the separation matrix in BSS, and of $f(\mathbf{A})=\sum_{1 \leq i \neq j \leq M}\left|a_{i j}\right|^{2}=$ $\|$ of $f(\mathbf{A}) \|_{F}^{2}$, where $\mathbf{A}$ is an $M \times M$ matrix. Here, we try to use the same notation as stated in Section III. This criterion is simple to implement and can be theoretically justified. The Jacobi method, the gradient method, and the Newton method can all be used to minimize this criterion [4], [25], [34], [55], [56]. These methods can also be classified into either of those works based on SOS, as in [34], or those works based on HOS, as in [25]; most of them consider the orthogonality constraint. Unlike this criterion, a log likelihood criterion proposed in [31] is more suitable for measuring the diagonality of a set of positive definite Hermitian matrices without the orthogonality constraint

$$
\mathcal{J}(\mathbf{W})=\underset{\mathbf{W}}{\operatorname{argmin}} \sum_{k=1}^{K} n_{k} \log \left(\frac{\operatorname{det} \operatorname{diag}\left(\mathbf{W}^{H} \overline{\mathbf{R}}_{k} \mathbf{W}\right)}{\operatorname{det}\left(\mathbf{W}^{H} \overline{\mathbf{R}}_{k} \mathbf{W}\right)}\right)
$$

where $n_{k}$ is a factor measuring the distribution of $\overline{\mathbf{R}}_{k}$. Another characteristic of this criterion is its invariance property regarding a scale change that is not held by the off-diagonal criterion. Recently, a new criterion based on the Cayley transform has been proposed in [57]. The criterion is performed in two steps:

$$
\mathcal{J}(\mathbf{W})=\underset{\left\{\mathbf{W}_{k}\right\}}{\operatorname{argmin}} \sum_{k=1}^{K}\left\|\mathbf{W}_{k}-\overline{\mathbf{W}}\right\|_{F}^{2}
$$

where $\mathbf{W}_{k}$ are the different unitary diagonalizers produced by eigendecomposition of covariance matrices for different time 
lags, and $\overline{\mathbf{W}}$ is the inverse Cayley transform of a weighted average matrix of the individual unitary matrices. The similarity between these two matrices provides a reasonable measure for joint diagonalization since different unitary matrices, with identical dimensions, are equivalent. More recently, an interesting linear criterion was proposed in [32]

$$
\mathcal{J}(\mathbf{W})=\underset{\mathbf{W}}{\operatorname{argmin}}\left\{-\sum_{k=1}^{K} \operatorname{tr}\left[C_{k}\left(\mathbf{W}^{H} \overline{\mathbf{R}}_{k} \mathbf{W}\right)\right]\right\}
$$

where $C_{k}=\operatorname{diag}\left(c_{1}^{(k)}, \ldots, c_{1}^{(k)}\right), k=1, \ldots, K$ are constant diagonal matrices, which can be adjusted to have similar orders as the diagonal elements of $\mathbf{W}^{H} \overline{\mathbf{R}}_{k} \mathbf{W}$ so that the effect of closely spaced eigenvalues of the matrices to be jointly diagonalized can be canceled. However, due to the order of diagonal elements of $\mathbf{W}^{H} \overline{\mathbf{R}}_{k} \mathbf{W}$ at critical points being unknown a priori, this criterion cannot work by itself.

As will be elaborated in Section VI, however, the different joint diagonalization criteria, considering the case either with orthogonal (unitary) constraints $\mathbf{W} \mathbf{W}^{H}=\mathbf{I}$ as in [21], [25], [32], and [57], or with nonorthogonal (nonunitary) constraints $\mathbf{W} \mathbf{W}^{H} \neq \mathbf{I}$, as in [4], [31], and [33], can be unified by using the penalty function (see Section V-A) as an unconstrained optimization problem. This makes it easier for us to carry out the theoretical analysis for the behavior of the joint diagonalization criterion for BSS problems, where the penalty function can be appropriately chosen to comply with the orthogonal and the nonorthogonal constraints. After such processing, to minimize the cost function in (16) (see Section V-A), we can resort to any unconstrained optimization algorithms such as the gradient-based (including steepest descent and conjugate gradient) methods, (quasi-)Newton methods, or any other alternatives.

\section{B. Constraints on BSS}

Generally, BSS employs the least possible information pertaining to the sources and the mixing system. However, in practice, there exists useful information to enhance the separability of the mixing system, which can be exploited to generate various effective algorithms for BSS. For example, a constrained parameter space specified by a geometric structure with $\|\mathbf{w}\|=1$ has been exploited in [45], where the norm restriction of the separation matrix is necessary for practical hardware implementations. The algorithms for adapting $\mathbf{W}(k)$ within the space of orthonormal matrices i.e., $\mathbf{W}(k) \mathbf{W}^{T}(k)=\mathbf{I}$, were first proposed in [48] and [49] and then developed in [46]. A merit of such algorithms lies in the possibility of extracting an arbitrary group of sources. This idea has also been addressed as the optimization problem on the Stiefel manifold or the Grassman manifold in [21], [38], [46], [50], and [51]. The key principle of such methods is to exploit the geometry of the constraint surface to develop algorithms searching either along a geodesic (the shortest line between two points on a curve surface) [51] or other paths [21]. In [52], the natural gradient procedures were developed to maintain a nonholonomic constraint on $\mathbf{W}(k)$. The geometric information of sources was considered in [44] as a constraint on BSS. Recently, a non-negative constraint on BSS has been shown to possibly be a useful way to represent real-world data [54]. This brief overview, together with a recent contribution to a similar work in [53], justifies that imposing an appropriate constraint on the separation matrix $\mathbf{W}(k)$ or the estimated source signals with special structure, such as invariant norm, orthogonality, geometry information, or non-negativity, provides meaningful information to develop a more effective BSS solution, especially for real-world signals and practical problems.

\section{Penalty Function-Based Joint DiAGONALIZATION APPROACH}

\section{A. Approach}

Constrained optimization problems can be effectively transformed into unconstrained ones by using the penalty function method, which adds a penalty term to represent constraint violation within the objective function [23], [24], [42]. A penalty function is a non-negative function, which is zero within the feasible area and positive within the infeasible area. A feasible point corresponds to one where all the constraints are satisfied. Typical constrained optimization problems are either subject to an equality constraint, an inequality constraint, or both. In this paper, we are particularly interested in the former type, due to the fact that the most general applications in BSS are subject to an equality constraint. Recalling a standard nonlinear equality constraint optimization problem in [24] (also see [23]) and extending the idea to the optimization for matrices, we have the following equality constraint optimization problem:

$$
\min f(\mathbf{W}) \text { s.t. } \mathbf{g}(\mathbf{W})=\mathbf{0}
$$

where $\mathbf{g}(\mathbf{W})=\left[g_{1}(\mathbf{W}), g_{2}(\mathbf{W}), \ldots, g_{r}(\mathbf{W})\right]^{T}: \mathbb{C}^{N \times M} \rightarrow$ $\mathbb{R}^{r}, f(\mathbf{W}): \mathbb{C}^{N \times M} \rightarrow \mathbb{R}^{1}, \mathbf{W} \in \mathbb{C}^{N \times M}$, and $r \geq 1$ indicates that there may exist more than one constraint. Let

$$
\begin{aligned}
\breve{\mathbf{w}} & =\operatorname{vec}(\mathbf{W}) \\
& =\left[w_{11}, \ldots, w_{N 1}, w_{12}, \ldots, w_{N 2}, \ldots, w_{1 M}, \ldots, w_{N M}\right]^{T}
\end{aligned}
$$

so that $\breve{\mathbf{w}} \in \mathbb{C}^{N M}$. This means that problem (10) can be reformulated as $\min f(\breve{\mathbf{w}})$, s.t. $\mathbf{g}(\breve{\mathbf{w}})=\mathbf{0}$. This is equivalent to the standard formulation in [24], which can, on the other hand, be deemed as a case for (10) by letting $\mathbf{W} \in \mathbb{C}^{N \times 1}$ and will be further utilized in Section VI. The transformation between the vector form and matrix form in the derivation of the algorithms can be followed as in [40]. In this paper, the complex-valued case is considered for generality. This is simply done by considering the real and imaginary parts simultaneously.

In the BSS context, $f(\mathbf{W})$ can be reformulated following any of the joint diagonalization criteria in Section IV-A, and $\mathbf{g}(\mathbf{W})$ can take the form of any constraint in Section IV-B. Penalty functions can be classified into two categories: exterior and interior. For the equality constraint problem (10), an exterior penalty function fits best due to the fact that interior penalty functions can only be used for sets defined by inequality constraints. We next give a self-contained definition of the exterior penalty function below.

Definition 1: Let $\mathcal{W}$ be a closed subset of $\mathbb{C}^{N \times M}$. A sequence of continuous functions $\mathcal{U}_{q}(\mathbf{W}): \mathbb{C}^{N \times M} \rightarrow \mathbb{R}^{1}, q \in \mathbb{N}$ 
is a sequence of exterior penalty functions if the following three conditions are satisfied:

$$
\begin{aligned}
\mathcal{U}_{q}(\mathbf{W}) & =0, \quad \forall \mathbf{W} \in \mathcal{W}, q \in \mathbb{N} \\
0 & <\mathcal{U}_{q}(\mathbf{W})<\mathcal{U}_{q+1}(\mathbf{W}) \\
\forall \mathbf{W} & \notin \mathcal{W}, q \in \mathbb{N} \\
\mathcal{U}_{q}(\mathbf{W}) & \rightarrow \infty, \text { as } \quad q \rightarrow \infty, \quad \forall \mathbf{W} \notin \mathcal{W} .
\end{aligned}
$$

A diagramatical representation of such functions is shown in Fig. 1.

Suppose that $\mathbf{U}(\mathbf{g}(\mathbf{W}))$ is a real-valued non-negative penalty function vector corresponding to the constraints $\mathrm{g}(\mathbf{W})$, i.e., $\mathbf{U}(\mathbf{g}(\mathbf{W}))=\left[\mathcal{U}_{1}\left(g_{1}(\mathbf{W})\right), \mathcal{U}_{2}\left(g_{2}(\mathbf{W})\right), \ldots, \mathcal{U}_{r}\left(g_{r}(\mathbf{W})\right)\right]^{T}$, and that $\boldsymbol{\xi}=\left[\xi_{1}, \xi_{2}, \ldots \xi_{r}\right]^{T}$ is a coefficient vector that controls the penalized values for the corresponding constraints; then, by using penalty functions, (10) is changed to

$$
\min \left\{f(\mathbf{W})+\boldsymbol{\xi}^{T} \mathbf{U}(\mathbf{g}(\mathbf{W}))\right\}
$$

An important issue for the successful implementation of the penalty function method is to choose a proper penalty function for the cost function since not all penalty functions ensure the minimization of the cost function [43]. In general, there are different penalty functions that can be utilized to characterize different problems. It is unlikely that a generic penalty function exists that is optimal for all constrained optimization problems. In Section VI, two penalty functions are introduced for both the unitary and nonunitary constraint, respectively. These two penalty functions are quite suitable for our applications in terms of the theoretical basis given below in Lemma 1 and numerical experiments given in Section VII. However, it should be noted that for a practical application with desired purpose, it is difficult to define a generic scheme to guide the user in terms of choice of penalty functions, but our work demonstrates that practically useful schemes can be found. To better understand the following discussion, we consider the following lemma.

Lemma 1: Suppose that $\mathrm{g}(\mathbf{W}): \mathbb{C}^{N \times M} \rightarrow \mathbb{R}^{r}$ is continuous and that $\mathcal{W}:=\left\{\mathbf{W} \in \mathbb{C}^{N \times M} \mid \mathbf{g}(\mathbf{W})=\mathbf{0}\right\}$. For $q \in \mathbb{N}$, let $\zeta_{q}$ be such that $\zeta_{q+1}>\zeta_{q}>0$ and $\zeta_{q} \rightarrow \infty$, as $q \rightarrow \infty$, and let $\gamma \geq 1$. If we define $\mathcal{U}_{q}(\mathbf{W}): \mathbb{C}^{N \times M} \rightarrow \mathbb{R}$ as

$$
\mathcal{U}_{q}(\mathbf{W}) \triangleq \zeta_{q}\|\mathbf{g}(\mathbf{W})\|_{b}^{\gamma}
$$

where $b=1,2$, or $\infty$, then $\mathbb{C}^{N \times M} \rightarrow \mathbb{R}^{r}$ means that the exterior penalty function projects the space from one into another with a possibly different dimension. Then, $\mathcal{U}_{q}(\mathbf{W})$ will form a sequence of exterior penalty functions for the set $\mathcal{W}$.

We omit the proof of Lemma 1 since it is straightforward to verify it by following Definition 1 . The penalty function given in (15) forms a set of differentiable penalty functions that will be used in the following discussion.

Using a factor vector $\boldsymbol{\kappa}$ to absorb the coefficient $\boldsymbol{\xi}$ and incorporating exterior penalty functions (15), our novel general cost function becomes

$$
P_{2}: \mathfrak{J}(\mathbf{W}(\omega))=\mathcal{J}(\mathbf{W}(\omega))+\boldsymbol{\kappa}^{T} \mathbf{U}(\mathbf{W}(\omega))
$$

where $\mathbf{U}(\mathbf{W}(\omega))=\left[\mathcal{U}_{1}(\mathbf{W}(\omega)), \ldots, \mathcal{U}_{r}(\mathbf{W}(\omega))\right]^{T}$ is a set of penalty functions with desired properties that can be designed to meet our requirements, $\boldsymbol{\kappa}=\left[\kappa_{1}, \ldots, \kappa_{r}\right]^{T}$ $\left(\kappa_{i} \geq 0\right)$ are the weighting factors, and $\mathcal{J}(\mathbf{W}(\omega))$ represents a basic joint diagonalization criterion, such as

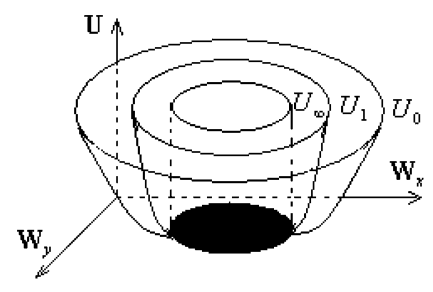

Fig. 1. $\mathcal{U}_{i}(\mathbf{W})(i=0,1, \ldots, \infty)$ are typical exterior penalty functions, where $\mathcal{U}_{0}(\mathbf{W})<\mathcal{U}_{1}(\mathbf{W})<\cdots<\mathcal{U}_{\infty}(\mathbf{W})$ and the shadow area denotes the subset $\mathcal{W}$.

in (8). In an expansion form, the criterion is denoted by $\mathfrak{J}(\mathbf{W}(\omega))=\mathcal{J}(\mathbf{W}(\omega))+\sum_{i=1}^{r} \kappa_{i} \mathcal{U}_{i}(\mathbf{W}(\omega))$. The separation problem is thereby converted into a new unconstrained joint diagonalization problem, i.e., $\underset{\mathbf{W}}{\operatorname{argmin}} \mathfrak{J}(\mathbf{W}(\omega))$.

Minimization of $\mathfrak{J}(\mathbf{W}(\omega))$ is approximately equivalent to the problem of minimization of $\mathcal{J}(\mathbf{W})$. However, note that in fact, the solution to problem (10) does not guarantee a solution for problem $P_{2}$. Only when $\mathbf{U}(\mathbf{W}(\omega))$ represents a set of exact penalty functions for (10) are the solutions equivalent [24]. Incorporating penalty functions into a joint diagonalization criterion enables us to convert constrained joint diagonalization problems into unconstrained ones and unify several methods using joint diagonalization for BSS; it can be used to construct special algorithms (including globally stabilizing locally converging Newton-like algorithms) for joint diagonalization problems. The constraint $\mathbf{W}(\omega) \neq \mathbf{0}$ in (8) can be easily satisfied by choosing a suitable penalty function $\mathcal{U}_{i}(\mathbf{W}(\omega))$ in (16) so that the degenerate solution can be automatically removed. As will be revealed in Sections V-B and VII, a proper penalty function may adapt the separation coefficients in a more uniform way, which generally indicates a better convergence performance (also see [18]).

Assuming that the penalty functions are in the form of (15), e.g., $\mathcal{U}_{i}(\mathbf{W}(\omega))=\|\mathbf{g}(\mathbf{W})\|^{2}$, and $\boldsymbol{\sigma}(\omega)$ is the vector of perturbations of $\mathbf{g}(\mathbf{W})$, where $\boldsymbol{\sigma}(\omega)=\left(\sigma_{1}(\omega), \ldots, \sigma_{r}(\omega)\right)^{T}$, then minimization of the criterion with the equality constraint $\mathrm{g}(\mathbf{W})=\mathbf{0}$ is equivalent to

$$
\begin{aligned}
\min _{\mathbf{W}} \mathfrak{J}(\mathbf{W}(\omega))= & \min _{\boldsymbol{\sigma}}\left\{\kappa\|\boldsymbol{\sigma}(\omega)\|^{2}\right\} \\
& +\min _{\mathbf{W}}\{\mathcal{J}(\mathbf{W}(\omega)): \mathbf{g}(\mathbf{W})=\boldsymbol{\sigma}\} \\
= & \min _{\boldsymbol{\sigma}}\left\{\kappa\|\boldsymbol{\sigma}(\omega)\|^{2}+\vartheta(\boldsymbol{\sigma})\right\}
\end{aligned}
$$

where $\vartheta(\boldsymbol{\sigma})$ is the perturbation function defined as the optimal value function for the equality constraint problem. Equation (17) implies that by adding the term $\kappa\|\boldsymbol{\sigma}(\omega)\|^{2}$, an attempt is made to convexify $\vartheta(\boldsymbol{\sigma})$ as $\kappa$ increases, and as $\kappa \rightarrow \infty$, the perturbation value $\boldsymbol{\sigma}$ approaches zero. This indicates that by increasing the value of the penalty parameter, the penalty function-based criterion has the ability to self-adjust in the presence of the perturbation. Similar discussions are given to show the stability of the algorithms for unitary and nonunitary constraints in Section VI.

It is worth noting that the criterion (16) has a similar form as that of exploiting well-known Lagrangian multipliers; however, they are essentially two different approaches for the constrained optimization problem. Using the penalty function, the 
solution to (14) approaches the same solution to (10) while satisfying the constraint simultaneously. When the penalty coefficients $\boldsymbol{\xi} \rightarrow \infty, \mathbf{U}(\mathbf{g}(\mathbf{W})) \rightarrow \mathbf{0}$, and hence, $\mathbf{g}(\mathbf{W})=\mathbf{0}$ can be satisfied. For the nonlinearly constrained problem, local minimizers of (10) are not necessarily local minimizers of the Lagrangian-based cost function so that it is not possible to design consistent line search rules based on the Lagrangian function, which is nevertheless especially important for smoothly connecting global optimization techniques with an effective asymptotic procedure, e.g., [41]. In this case, a better choice is to construct a global strategy for problem (10) based on penalty functions. In addition, for a nonconvex problem, a penalty function-based method can tend to the optimal solution for the original problem with increasing penalty coefficients $\boldsymbol{\kappa}$; however, the Lagrangian approach would fail to produce an optimal solution of the primal problem due to the presence of a duality gap [23].

\section{B. Global Minima and Local Minima}

The equivalence between (16) and (10) follows from the results in [24], which provide the fundamental theoretical basis for our approach, including its global and local minima given by the following lemmas.

We define the sets $\mathcal{W}:=\left\{\mathbf{W} \in \mathbb{C}^{N \times M} \mid \mathbf{g}(\mathbf{W})=\mathbf{0}\right\}$ and $\mathcal{B}(\hat{\mathbf{W}}, \rho):=\left\{\mathbf{W} \in \mathbb{C}^{N \times M} \mid\|\mathbf{W}-\hat{\mathbf{W}}\|_{F} \leq \rho\right\}$, where $\rho>0$, and we assume the following: A1) $\exists \mathbf{W}^{*}$ s.t. $\mathcal{A}:=$ $\left\{\mathbf{W} \in \mathbb{C}^{N \times M} \mid f(\mathbf{W}) \leq f\left(\mathbf{W}^{*}\right)\right\}$ is compact, and A2) $\hat{\mathbf{W}}=$ $\arg \min f(\mathbf{W})$ s.t. $\mathbf{W} \in \mathcal{B}(\hat{\mathbf{W}}, \rho) \cap \mathcal{W} \neq \varnothing$. As is convention

with the use of penalty functions in nonlinear optimization [24], the $i$ th trial, where $i \in \mathbb{N}$, corresponds to one setting of a scalar penalty coefficient, which is denoted $\kappa^{i}$, and the accumulation point is the minimizer $\hat{\mathbf{W}}$ when $\kappa^{i} \rightarrow \infty$. Then, we have two lemmas.

Lemma 2: If $\mathbf{W}_{i}$ is the minimizer of $P_{2}$ at the $i$ th trial, then in the limit, as $\kappa^{i} \rightarrow \infty, \mathbf{W}_{i} \rightarrow \hat{\mathbf{W}}$, the accumulation point is as in assumption A2.

Lemma 3: If $\mathbf{W}_{i}, \forall i$ is a strict local minimizer of $P_{2}$ at the $i$ th trial, i.e., for some $\rho_{i}>0, f_{i}\left(\mathbf{W}_{i}\right)<f_{i}(\mathbf{W}), \forall \mathbf{W} \in$ $\mathcal{B}\left(\mathbf{W}_{i}, \rho_{i}\right):=\left\{\mathbf{W} \in \mathbb{C}^{N \times M}\left\|\mid \mathbf{W}-\mathbf{W}_{i}\right\|_{F} \leq \rho_{i}\right\}$, then in the limit as $\kappa^{i} \rightarrow \infty$, there is an accumulation point $\hat{\mathbf{W}}$, as in assumption A2, for which $\exists \rho>0$, s.t. $\rho_{i} \geq \rho, \forall i \in \mathbb{N}$, and $\hat{\mathbf{W}}$ is a local minimizer of (10).

Assumption A1 ensures that problem (10) has a solution, and A2) ensures that the closure of the set $\mathcal{B}(\hat{\mathbf{W}}, \rho) \cap \mathcal{W}$ contains an optimal solution to problem (10). The lemmas imply that the new criterion (16) holds the same global and local properties as that without the penalty term when given large enough penalty parameters. This means that the choice of the penalty parameters usually has a major effect on the overall optimization accuracy in practical implementation. Too small parameters will lead to an inexact or even incorrect final solution, whereas too large values may create a computationally poorly conditioned or strongly nonlinear energy function [43]. Unlike the standard penalty function method ultimately requiring infinite penalty parameter $\kappa^{i}$, in practice, with time-varying signals, we employed a finite $\kappa^{i}$ to obtain a performance advantage as supported by our simulation results. A similar technique is used in [39] for blind detection of desired signals from multiple chan- nels in a direct-sequence code division multiple access system. This idea is based on the following corollary [24].

Corollary 1: If $\mathcal{U}_{i}\left(g_{i}\left(\mathbf{W}_{i}\right)\right)=0$ for some $\kappa^{i}$, then $\mathbf{W}_{i}$ is an optimal solution to the problem (10).

Therefore, in our practical application, we can relax the theoretical condition that $\kappa^{i} \rightarrow \infty$, provided $\mathcal{U}_{i}\left(g_{i}\left(\mathbf{W}_{i}\right)\right) \approx 0$, and attain performance advantage with finite $\kappa^{i}$. This also mitigates another problem in implementation of the penalty function method, i.e., that the introduction of penalties may transform a smooth objective function into a rugged one. To avoid local minima in the objective function, the penalized joint diagonalization cost function must preserve the topology of the objective function to allow location of the optimal feasible solution; see [24] for more details.

\section{Penalized Joint Diagonalization With UNITARY/NONUNITARY CONSTRAINT}

\section{A. BSS With Unitary and Nonunitary Constraint}

For unitary constraint, the problem is formulated as the minimization of a cost function $\mathcal{J}(\mathbf{W})$ under the constraint $\mathbf{W} \mathbf{W}^{H}=\mathbf{I}$. That is, $\mathcal{J}(\mathbf{W})$ is a function of the subspace spanned by the columns of $\mathbf{W}$. It has been shown that there are several potential approaches to solving the unitary constrained BSS problem, including the whitening approach (e.g., [25], [34], and [37]), projection on the Stiefel-Grassman manifold (e.g., [5], [21], [38], and [46]), and other approaches (e.g., [33] and [35]). The algorithms were developed with the aim of restricting the separation matrix to be orthogonal. From the discussion in Section IV-B, the constrained minimization of a cost function is converted into the unconstrained optimization on a manifold. Particularly, the orthogonal constrained optimization problem in [21] can be treated as a special case of the unconstrained problem in (16) so that Lemma 1 is applicable for such a case. This idea can find a number of applications in the areas of signal processing and linear algebra, such as joint diagonalization, subspace tracking, or singular value decomposition [5], [25], [29], [34], [35].

However, the methods using unitary constraint may result in a degraded performance in the context of BSS, due to the unbalanced weighting of the misdiagonalization between all the matrices being jointly diagonalized. The nonunitary methods were therefore addressed in a more general way [18], [22], [30], [31], [33]. A common characteristic of the works in [22], [31], and [30] is that no hard whitening is exerted on the separation process. In [33], a combination of several possible cost functions was proposed for BSS; however, no details were found to address the behavior and performance of such combinations. In [18], the idea of using penalty functions for nonunitary constraint with application to frequency-domain BSS was presented. This work, regardless of initialization, and similar to that in [22], is different from [30], which is typical for Gauss-Newton-type algorithms and needs the separation matrix to be properly initialized.

\section{B. Unifying by Penalty Function With Perturbation Analysis}

Compared with the methods discussed in Section VI-A, which all considered instantaneous mixtures in the time do- 
main, the method addressed here operates in the frequency domain. Incorporating the penalty function discussed earlier, the total cost function becomes

$$
\mathfrak{J}(\mathbf{W})=\underset{\mathbf{W}}{\operatorname{argmin}} \sum_{\omega=1}^{T} \sum_{k=1}^{K}\{\mathcal{F}(\mathbf{W})(\omega, k)+\kappa \mathcal{G}(\mathbf{W})(\omega, k)\}
$$

where $\kappa$ is a penalty weighting factor.

The key problem is now to choose a suitable penalty function. For a unitary constraint and a nonunitary constraint, the alternative but equivalent forms can be chosen as $\| \mathbf{W}(\omega) \mathbf{W}^{H}(\omega)-$ $I \|_{F}^{2}$ (see [46]) and $\|\operatorname{diag}(\mathbf{W}(\omega)-I)\|_{F}^{2}$ (see [33]). If we choose the penalty function to be in the form of $\left\|\mathbf{W}(\omega) \mathbf{W}^{H}(\omega)-I\right\|_{F}^{2}$ or $\|\operatorname{diag}(\mathbf{W}(\omega)-I)\|_{F}^{2}$, we will see that using a penalty function with a limited norm will control the size of the designed parameters. This property would be especially useful when the cost function is not explicitly dependent on the design parameters, such as $\mathbf{W}(\omega)$, where the case may happen in some optimization problems that are ill-posed in the sense that solutions with bounded controls do not exist. Note that the compact assumption A1 in Lemma 2 and 3 is addressed with respect to the objective function $\mathcal{F}(\mathbf{W})(\omega, k)$ in (18), regardless of the form of the constraint, e.g., $\mathbf{W}(\omega) \mathbf{W}^{H}(\omega)=I$. In addition, even if $\mathbf{W}(\omega)$ is complex valued, assumption a) is still satisfied due to the real-valued penalty function being used.

Under the penalty function-based framework, either a unitary or a nonunitary constraint problem can be deemed as an example of a penalized unconstrained optimization problem, whereas the forms of the penalty functions may be different. We may choose a penalty function with the form of $\mathcal{G}(\mathbf{W})=\mathbf{g}(\mathbf{W})^{T} \mathbf{g}(\mathbf{W})$ (see [39] for a similar application) or $|\operatorname{Tr}[\mathbf{g}(\mathbf{W}) \tilde{\Lambda}]|$ (see [36] for a similar application), where $\tilde{\Lambda}$ is a diagonal matrix containing the Lagrangian multipliers.

It would be useful to examine the eigenvalue structure of the Hessian matrix of the cost function, i.e., $\nabla^{2} \mathfrak{J}$, which dominates the convergence behavior of the algorithms used for minimizing $\mathfrak{J}(\mathbf{W})$. Following a similar procedure as in Appendix A, by calculating the perturbation matrix $\Delta$ of $\mathbf{W}$ and assuming that the cost function is twice-differentiable

$$
\begin{aligned}
\nabla^{2} \mathcal{F}(\mathbf{W})= & 4\left(\left[\mathbf{R}_{\mathbf{X}}-\mathbf{R}_{\mathbf{V}}\right] \otimes \operatorname{off}\left(\mathbf{R}_{\mathbf{Y}}\right)\right) \\
& +4\left(\left[\mathbf{R}_{\mathbf{X}}-\mathbf{R}_{\mathbf{V}}\right]^{T} \mathbf{W}^{T} \otimes \mathbf{I}\right) \\
& \times \mathbf{D}\left(W^{*}\left[\mathbf{R}_{\mathbf{X}}-\mathbf{R}_{\mathbf{V}}\right]^{*} \otimes \mathbf{I}\right)
\end{aligned}
$$

where $\otimes$ is the Kronecker product, $\mathbf{D}=\operatorname{diag}(\mathrm{vec}$ $\left.\left(\operatorname{off}\left(\mathbf{1}^{M \times M}\right)\right)\right)$, and $\mathbf{1}^{M \times M}$ is a matrix whose elements are all ones

$$
\begin{aligned}
\boldsymbol{\nabla}^{2}[\kappa \mathcal{G}(\mathbf{W})]=\kappa \frac{\partial \mathcal{G}(\mathbf{W})}{\partial \mathbf{W}^{*}} & \nabla^{2} g_{i}(\mathbf{W}) \\
& +\kappa \frac{\partial^{2} \mathcal{G}(\mathbf{W})}{\partial \mathbf{W}^{*}} \nabla g_{i}(\mathbf{W}) \boldsymbol{\nabla} g_{i}(\mathbf{W})^{T}
\end{aligned}
$$

The $\nabla^{2} \mathfrak{J}$ is the linear combination of $\nabla^{2} \mathcal{F}(\mathbf{W})$ and $\nabla^{2}[\kappa \mathcal{G}(\mathbf{W})]$. The conditions of Lemma 2 indicate that as $\kappa \rightarrow \infty, \mathbf{W}$ will approach the optimum $\hat{\mathbf{W}}$. If $\hat{\mathbf{W}}$ is a regular solution to the constrained problem, then there exist unique Lagrangian multipliers $\bar{\lambda}_{i}$ such that $\left(\partial \mathcal{G}(\hat{\mathbf{W}}) / \partial \mathbf{W}^{*}\right)+\sum \bar{\lambda}_{i} \boldsymbol{\nabla} g_{i}(\mathbf{W})=\mathbf{0}$ [23]. This means that $\kappa\left(\partial \mathcal{G}(\mathbf{W}) / \partial \mathbf{W}^{*}\right) \rightarrow \bar{\lambda}_{i}$ as $\mathbf{W} \rightarrow \hat{\mathbf{W}}$. Combining (19) and (20), we have

$$
\begin{aligned}
\nabla^{2} \mathfrak{J}(\mathbf{W}) \triangleq \nabla^{2} \mathcal{F}(\mathbf{W}) & +\kappa \frac{\partial \mathcal{G}(\mathbf{W})}{\partial \mathbf{W}^{*}} \nabla^{2} g_{i}(\mathbf{W}) \\
& +\kappa \frac{\partial^{2} \mathcal{G}(\mathbf{W})}{\partial \mathbf{W}^{*}} \nabla g_{i}(\mathbf{W}) \nabla g_{i}(\mathbf{W})^{T} .
\end{aligned}
$$

The first two terms on the right-hand side of (21) approach the Hessian of the Lagrangian function $L(\mathbf{W})=\mathcal{F}(\mathbf{W})+$ $\sum \bar{\lambda}_{i} g_{i}(\mathbf{W})$. The last term in (21) strongly relies on $\kappa$. It can be shown that as $\kappa \rightarrow \infty, \nabla^{2} \mathfrak{J}(\mathbf{W})$ has some eigenvalues approaching $\infty$, depending on the number of the constraints, and the other eigenvalues approach finite value. The infinite eigenvalues reveal the reason behind the ill-conditioned computation problem mentioned in Section V-B. Letting $\epsilon$ be the step size in the adaptation, perturbation of the cost function in the iterative update is then $\mathcal{F}(\mathbf{W}+\epsilon \boldsymbol{\Delta})+\kappa \mathcal{G}(\mathbf{W}+\epsilon \boldsymbol{\Delta})$. In the presence of nonlinear equality constraints, the direction $\Delta$ may cause any reduction of $\mathcal{F}(\mathbf{W}+\epsilon \boldsymbol{\Delta})$ to be shifted by $\kappa \mathcal{G}(\mathbf{W}+\epsilon \boldsymbol{\Delta})$. This needs the step size to be very small to prevent the ill-conditioned computation problem induced by large eigenvalues at the expense of having a lower convergence rate.

\section{Gradient-Based Adaptation}

In this section, we develop a gradient-based descent method to adapt the coefficients in (18). The method is actually a least squares (LS) estimation problem. Although the convergence rate of the gradient method is linear as compared to the Newton-type adaptation method, which can achieve quadratic convergence by using second-order derivatives, a gradient-based adaptation method usually has the ability to converge to a local minimum, whereas no guarantee exists for a Newton-type method, which may converge to the nearest critical point. One of the other advantages of the gradient-based adaptation method is its simplicity of implementation as well as its lower computational complexity.

Let the penalty function be $\mathcal{G}(\mathbf{W})(\omega, k)=$ $\left\|\mathbf{W}(\omega) \mathbf{W}^{H}(\omega)-\mathbf{I}\right\|_{F}^{2}$ in (18). For a unitary constraint (see [33] and [46]), the gradients in (18) with respect to their parameters can be readily derived as (see Appendix A)

$$
\begin{aligned}
\frac{\partial \mathfrak{J}}{\partial \mathbf{W}^{*}(\omega)}=4 \sum_{k=1}^{K}\{ & {\left[\operatorname{off}\left(\mathbf{R}_{Y}(\omega, k)\right)\right] \mathbf{W}(\omega) } \\
& \times\left[\mathbf{R}_{X}(\omega, k)-\mathbf{R}_{V}(\omega, k)\right] \\
& \left.+\kappa \mathbf{W}(\omega)\left[\mathbf{W}^{H}(\omega) \mathbf{W}(\omega)-\mathbf{I}\right]\right\} \\
\frac{\partial \mathfrak{J}}{\partial \mathbf{R}_{V}^{*}(\omega, k)}=-4 \sum_{k=1}^{K} \operatorname{diag}\left\{\mathbf { W } ^ { H } ( \omega ) \left[\mathbf{R}_{Y}(\omega, k)\right.\right. & \left.\left.-\operatorname{diag}\left(\mathbf{R}_{Y}(\omega, k)\right)\right] \mathbf{W}(\omega)\right\} .
\end{aligned}
$$

Theoretically, incorporating the penalty term $\| \mathbf{W}(\omega) \mathbf{W}^{H}(\omega)-$ $\mathbf{I} \|_{F}^{2}$ is equivalent to applying a projection operation on the gradient of $\mathcal{J}(\mathbf{W})(\omega, k)$; however, even for the latter method, the penalty function is useful to attain a stable performance [59]. 
For a nonunitary constraint, the adaptation is parallel to the foregoing procedure. Letting the penalty function be in the form of $\mathcal{G}(\mathbf{W})(\omega, k)=\|\operatorname{diag}[\mathbf{W}(\omega)-\mathbf{I}]\|_{F}^{2}$ (see [33]), the adaptation equations to find the coefficients in (18), corresponding to (22), become (see Appendix A)

$$
\begin{array}{r}
\frac{\partial \mathfrak{J}}{\partial \mathbf{W}^{*}(\omega)}=2 \sum_{k=1}^{K}\left\{2 [ o f f ( \mathbf { R } _ { Y } ( \omega , k ) ) ] \mathbf { W } ( \omega ) \left[\mathbf{R}_{X}(\omega, k)\right.\right. \\
\left.\left.-\mathbf{R}_{V}(\omega, k)\right]+\kappa \operatorname{diag}[\mathbf{W}(\omega)-\mathbf{I}]\right\} .
\end{array}
$$

In this case, (23) takes the same form. It is worth noting that an alternative adaptation method to (24) is to use a modified gradient by using a Lagrangian multiplier, which can adapt $\mathbf{W}(\omega)$ in a more uniform way; see [18] for more details.

Note that theoretically, when we update the algorithm by using rules (22) and (24), after it converges, the norm of $\mathbf{g}(\mathbf{W})$ in (15) approaches a finite value. When $\kappa \rightarrow \infty$, the multiplication will approach infinity in terms of Lemma 1 and, hence, satisfy (13). However, in practical implementation, (13) is exploited approximately. Such consideration is based on the following two reasons. First, there exist numerical problems in practical implementations. Second, when the penalty function approaches infinity, it will dominate the objective function, which, however, should not happen for a desired purpose. In our implementation, the cross-power spectrum matrix remains important in the objective function, and at the same time, the separation matrix satisfies a desired property to some degree.

Because the cross-correlation $\mathbf{R}_{X}(t, t+\tau)=E\{\mathbf{x}(t) \mathbf{x}(t+$ $\left.\tau)^{T}\right\}$ is time dependent for nonstationary signals, it is difficult to estimate the cross-power-spectrum with a relatively short stationary time resolution. However, in practice, $\mathbf{R}_{X}(\omega, k)$ can be estimated by the block form

$$
\hat{\mathbf{R}}_{X}(\omega, k)=\frac{1}{D} \sum_{m=0}^{D-1} \mathbf{X}(\omega, D k+m) \mathbf{X}^{H}(\omega, D k+m)
$$

where $D$ is the number of intervals used to estimate each cross power matrix. In implementation, the normalized step sizes for adaptation of $J_{M}(\mathbf{W})(\omega, k)$ and $J_{C}(\mathbf{W})(\omega, k)$ take the forms

$$
\begin{aligned}
\mu_{J_{M}}(\omega) & =\frac{\alpha}{\left(\sum_{k=1}^{K}\left\|\mathbf{R}_{X}(\omega, k)\right\|_{F}^{2}\right)} \\
\mu_{J_{C}}(\omega) & =\frac{\xi}{\left(\varsigma+\sum_{k=1}^{K}\left\|\frac{\partial J_{C}(\mathbf{W})(\omega, k)}{\partial \mathbf{W}^{*}(\omega)}\right\|_{F}\right)}
\end{aligned}
$$

where $\alpha, \xi$, and $\varsigma$ are scalar values adjustable for adaptation.

The LS solution to (18) can be obtained using the well-known stochastic gradient algorithm. The solution will be in the form of $\mathbf{W}^{(l+1)}(\omega)=\mathbf{W}^{(l)}(\omega)-\mu(\omega) \cdot\left(\partial \mathfrak{J} / \partial \mathbf{W}^{(l) *}(\omega)\right)$, where $(\cdot)^{*}$ denotes the complex conjugate operator.

\section{Scale and Permutation Indeterminacies}

There are possible indeterminacies in the model (1), such as sign, scale, spectral shape, and permutation [13], among which, the permutation ambiguity and the scale ambiguity are most important for the separation performance. In Section II, when we try to combine the results obtained at each frequency bin, the permutation problem occurs because of the inherent permutation ambiguity in the rows of $\mathbf{W}(\omega)$. Here, we use the method in [4] to address the permutation problem with regard to the filter length constraint of the FIR model so that we can compare the performance of the proposed method with other traditional techniques. One promising advantage of using the penalty function is its potential to reduce the scaling ambiguity. Taking the form of the unitary constraint in Section VI-C as an example, we see that by using the penalty function, not only the constraint tends to be satisfied, but the norm of the separation matrix is restricted to a desired value as well. This is especially useful in the iterative update, and the amplitude of the signal at each frequency bin is preserved to have normal energy. Such an effect on the scaling ambiguity while incorporating the penalty function will be demonstrated by a simulation in Section VII.

\section{E. Implementation Pseudo-Codes}

According to the discussions given in the above sections, taking the penalty functions with unitary or nonunitary constraints as examples, we summarize the whole algorithms as the following steps:

1) Initialize parameters $N, M, D, T, K$, $\mu, \kappa, \alpha, \varsigma, \xi, \mathbf{W}_{0}, \operatorname{IRN}$, and $\mathbf{W}_{0}(\omega)$.

2) Read input mixtures, i.e., time samples $\mathbf{x}(n)$ :

-For artificially mixing, $\mathbf{x}(n)$ is ob-

tained using (1).

-For the real mixing environment, $\mathbf{x}(n)$ is the signal recorded by a microphone array. 3) Calculate the cross-power spectrum matrix:

-Convert $\mathbf{x}(n)$ to $\mathbf{X}(\omega, n)$ using (2);

-Calculate $\mathbf{R}_{X}(\omega, k)$ using (25).

4) Calculate the cost function and update gradient:

$$
\begin{aligned}
& \text {-for } i=1 \text { to } I R N \\
& \text { * Update } \mu_{J_{M}}(\omega) \text { and } \mu_{J_{C}}(\omega) \text { using (26) and } \\
& \text { (27) respectively; } \\
& \text { * Update } \mathbf{W}(\omega) \leftarrow \mathbf{W}(\omega)+\mu \nabla \mathbf{W}(\omega) \text { using (22) } \\
& \text { or (24); } \\
& \text { * Update } \mathfrak{J}_{i}(\mathbf{W}(\omega)) \text { using (16) or (18); } \\
& \text { * if }\left(\mathfrak{J}_{i}(\mathbf{W}(\omega))>\mathfrak{J}_{i-1}(\mathbf{W}(\omega))\right) \text { break; } \\
& \text {-end. } \\
& \text { 5) Solve permutation problem } \mathbf{W}_{n e w}(\omega)
\end{aligned}
$$
$\mathcal{P}(\mathbf{W}(\omega))$, where $\mathcal{P}$ is a function dealing with permutation operation (refer to [4]). 6) Calculate $\mathbf{Y}(\omega, k)$ according to (4). Reconstruct the time domain signals $\mathbf{y}(n)=$ $\operatorname{IDFT}(\mathbf{Y}(\omega, k))$.

7) Calculate the performance index using

(29) or (31).

8) End. 


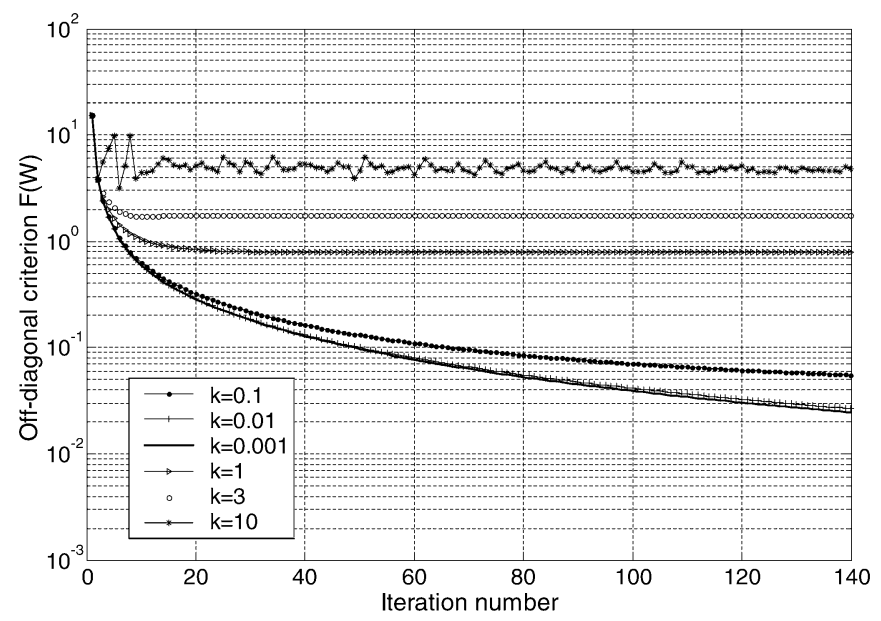

Fig. 2. Convergence performance of the cross-power spectrum based off-diagonal criterion changes under the penalty function constraint with various penalty coefficients $\kappa$.
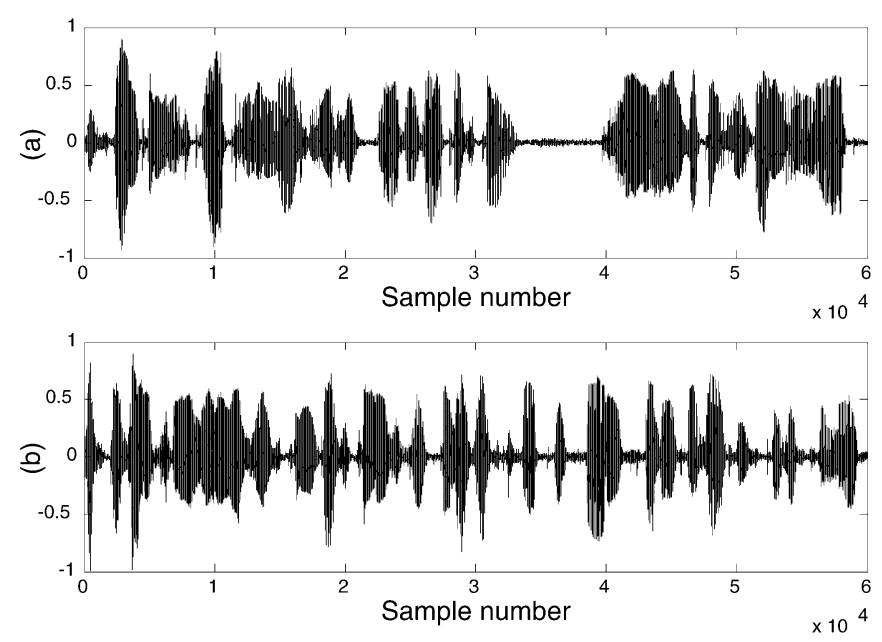

Fig. 3. Two source speech signals used in the experiment.

\section{NUMERICAL EXPERIMENTS}

In this section, we examine the performance of the proposed method by simulations when applied to both artificially mixed signals and real room recordings. The evaluations will be carried out in two aspects: convergence behavior and separation performance (see Fig. 2).

A system with two inputs and two outputs (TITO) is considered for simplicity, that is, $N=M=2$ (the simulations can be readily extended into MIMO systems due to the general formulation of the problem in the previous sections). The two source speech signals used in the following experiments are shown in Fig. 3 (sampling rate $12 \mathrm{kHz}$ ), which are available from [62]. We artificially mix the two sources by a nonminimum-phase system

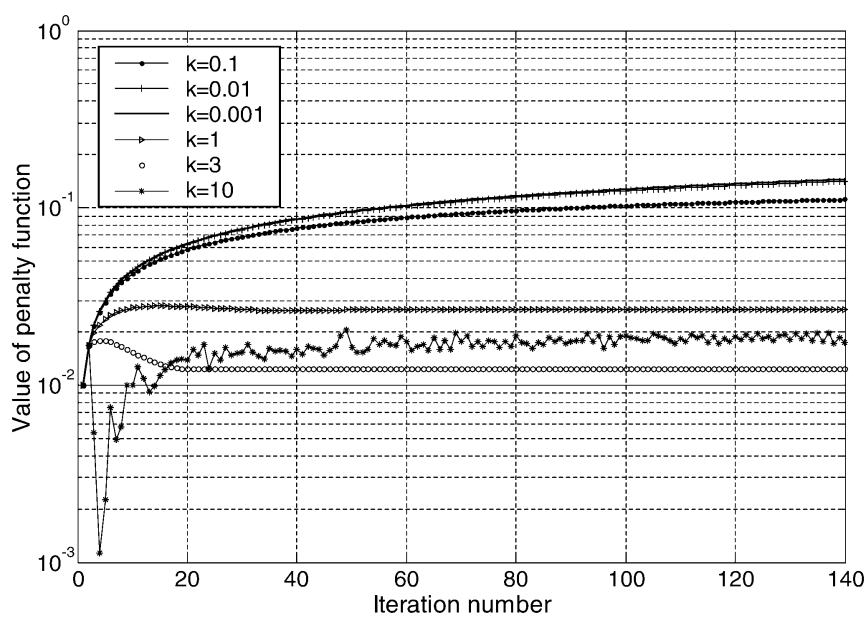

Fig. 4. Convergence performance of the penalty function with various penalty coefficients $\kappa$.

in (28) [47], shown at the bottom of the page. First, we investigate the convergence behavior of the proposed method by comparison with the method in [4], which corresponds to the case $\kappa=0$ in our formulation in the previous sections, and the following simulations were specified. We develop the work in [18] and choose the penalty function to be in the form of a nonunitary constraint (see also [59]). Adaptation (24) is therefore used to examine the convergence performance of the proposed method. The parameters are set as follows. The number of intervals used to estimate each cross-power-matrix is set to be $D=7$. The length of the fast Fourier transform (FFT) is $T=1024$. The parameters in the normalized step sizes given in (26) and (27) are set to be $\alpha=1, \varsigma=0.05$, and $\xi=0.2$, respectively. The number of the matrices to be diagonalized is $K=5$. The step size in gradient adaptation is $\mu=1$. The initial value of the separation matrix is $\mathbf{W}_{0}(\omega)=\mathbf{I}$. We applied the short term FFT to the separation matrix and the cross-correlation of the input data. Fig. 2 shows the behavior of the penalty coefficient $\kappa$ affecting the convergence of the cross-power spectrum based off-diagonal criterion. Fig. 4 shows how the penalty function itself changes with the penalty coefficients when the cross-power spectrum based off-diagonal criterion was constrained by the penalty function. From Figs. 2 and 4, we see that the separation algorithm generally has increasing convergence speed with the increasing value of the penalty coefficient $\kappa$, as well as a quicker trend approaching the constraint. An increase in the value of $\kappa$ introduces a stronger penalty being exerted to the off-diagonal criterion. This means, by increasing the penalty coefficient $\kappa$, that we may not only approach the constraint in a quicker way but also attain a better convergence performance. The convergence performance of the new criterion with penalty function, i.e., (18) is shown in Fig. 5, where the cost function converges to a lower value in a faster speed as compared with the

$$
\mathbf{H}(z)=\left[\begin{array}{cc}
1+1.0 z^{-1}-0.75 z^{-2} & 0.5 z^{-5}+0.3 z^{-6}+0.2 z^{-7} \\
-0.7 z^{-5}-0.3 z^{-6}-0.2 z^{-7} & 0.8-0.1 z^{-1}
\end{array}\right] .
$$




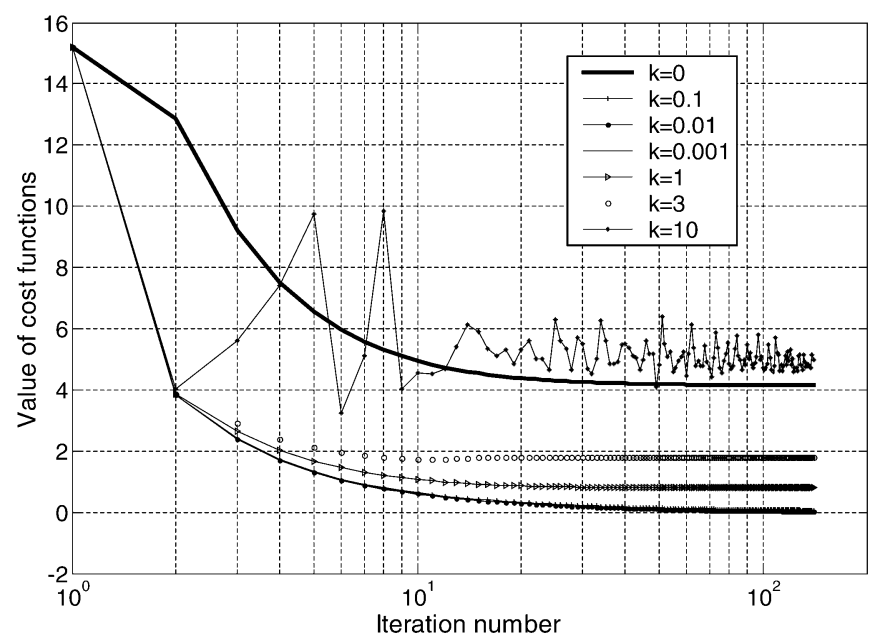

Fig. 5. Comparison of convergence performance of the new criterion (with penalty function constraint where $\kappa \neq 0$ ) and cross-power spectrum based off-diagonal criterion in [4] (without penalty function, where $\kappa=0$ ).

criterion in [4]. The convergence speed will be scaled by the penalty coefficient. The unusual behavior for $\kappa=10$ is due to ill-conditioning and will be discussed in more detail later. More precise examination of the convergence behavior is given in Fig. 6, where the adaptation stops when a threshold is satisfied, and the current value will be taken as the stable value of the criterion. From Fig. 6, it can be seen that by an appropriate choice of the penalty coefficient $\kappa$ (generally increasing $\kappa)$, we can obtain a significantly improved convergence speed, as well as a better performance, which is indicated by the lower level of the off-diagonal elements existing in the cross-correlation matrices of the output signals. Theoretically, increasing the value of the penalty coefficient will arbitrarily approach the constraint. However, as discussed in Sections V and VI, numerical problems will be induced due to large value of the penalty $\kappa$. Under a common step size, too large a penalty $\kappa$ will introduce ill-conditioned calculation, and this can be observed in Figs. 2, 4, and 5 for the case of $\kappa=10$, where there is fluctuation in the adaptation. At the same time, although a larger penalty will put higher emphasis on the constraint, the theoretical analysis in Section VI showed that the constraint itself has no necessary connection to the performance improvement. This can be confirmed in Fig. 6 (see $\kappa=10$ ). However, the property of performance improvement with increasing penalty $\kappa$ appears to be promising. An alternative method to fully exploit such a property is to decrease the step size $\mu$. Fig. 7 showed that the numerical ill-condition problem in Fig. 5 (when $\kappa=10$ ) can be removed by reducing the step size to a smaller value. This indicates that we can choose a suitably small step size to suppress the numerical problem. However, as shown in the case of $\mu=0.1$ and $\mu=0.01$ in Fig. 7, this means that a stable numerical performance for large penalties will be achieved at a small cost of convergence speed. Therefore, an optimal penalty coefficient will be obtained by a tradeoff consideration of the step size in practical implementations.

As discussed in Section VI-D, there are inherent permutation and scaling ambiguities in BSS algorithms. Here, we will show

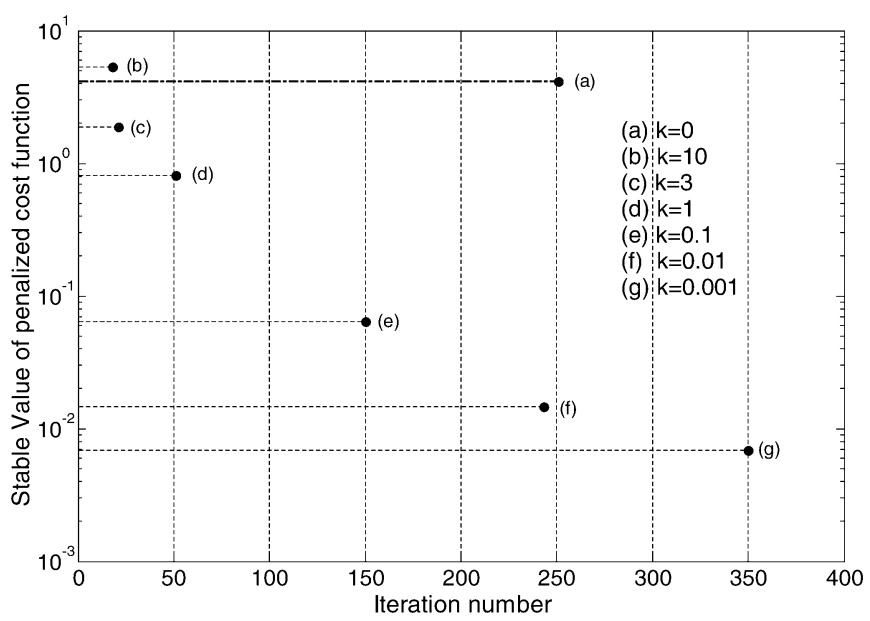

Fig. 6. Comparison of the stable values and the required iteration numbers (to reach such stable values) between the new criterion and the conventional cross-power spectrum-based criterion. (a) Corresponding to the criterion in [4] with $\kappa=0$. (b) $-(\mathrm{g})$ Corresponding to the new criterion with penalty coefficient $\kappa \neq 0$.

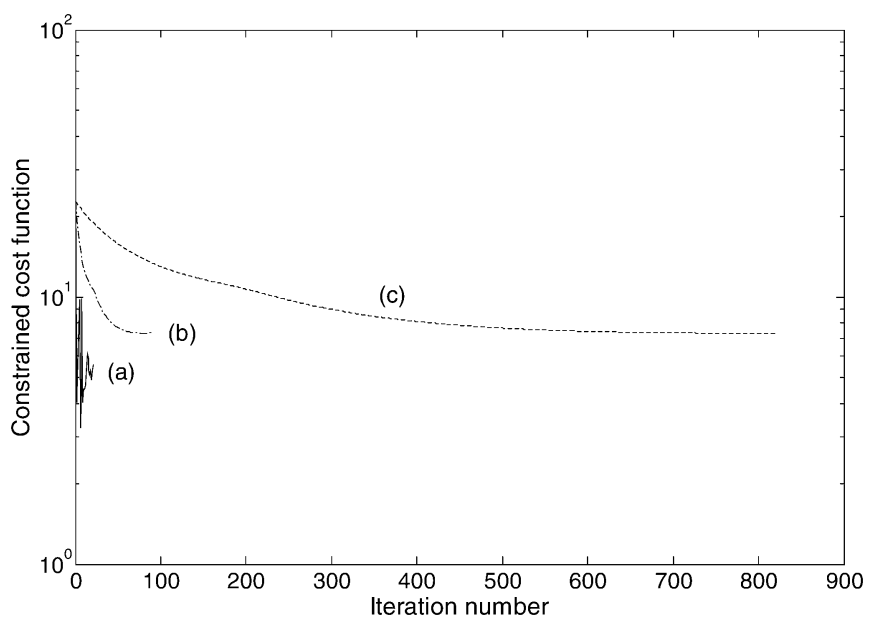

Fig. 7. Influence of step size on the penalty coefficients $\kappa$, where the step sizes $\mu$ in (a)-(c) are set to be $1,0.1$ and 0.01 , respectively, whereas the penalty coefficients $\kappa$ keep the same to be 10 . The adaptation stops when a threshold is satisfied, and the stable value of the cost function is obtained at this point.

the ability of the penalty function to suppress the scaling ambiguity by simulations and addressing the permutation problem, as in [4], to allow performance comparison. Theoretically, due to the independence assumption, the cross-correlation of the output signals in (5) should approximately approach zero. Fig. 8(a) shows the results using the off-diagonal criterion (8). From these results, we see that it is true at most frequency bins but not for very low frequency bins. From the remaining figures in Fig. 8, we see that such an effect can be significantly reduced using penalty functions by careful choice of the value of penalty coefficients used in the experiment, and the effect is almost removed when $\kappa$ is close to 0.01 in this case. It should be noted that the imaginary part is not plotted due to its similar behavior. An alternative to this problem can be found in [58], where such an effect was restricted by a normalization process using the diagonal elements of the cross-power matrices. 

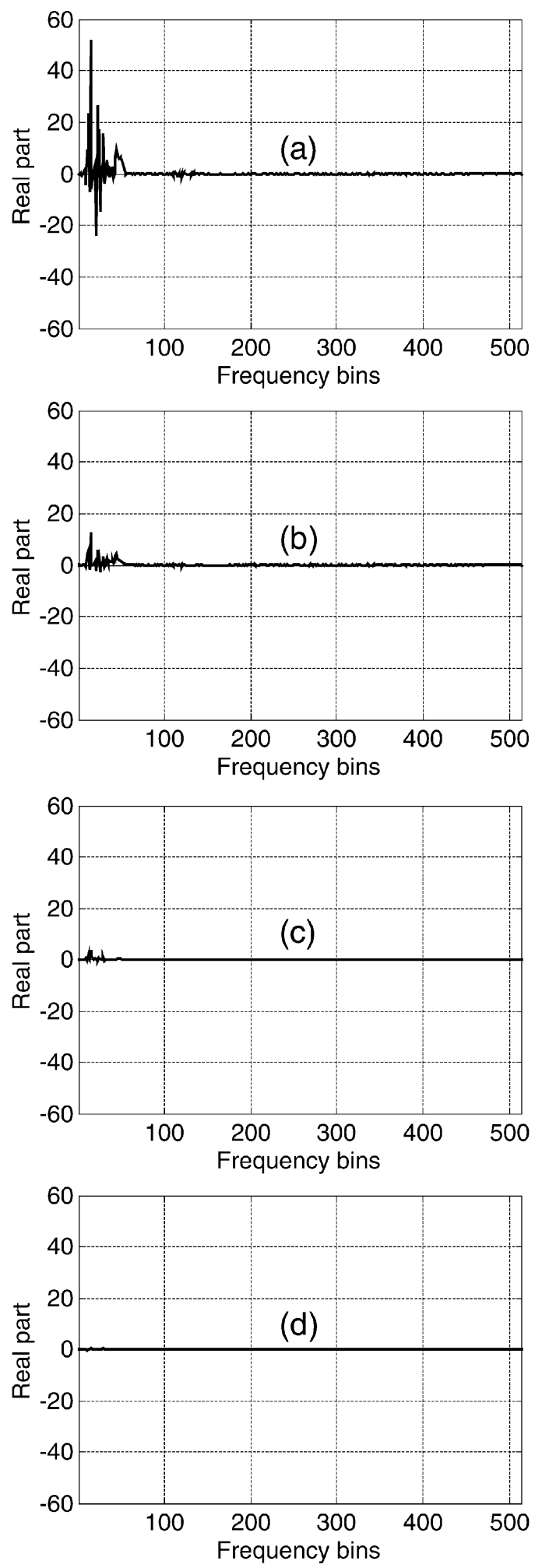

Fig. 8. Overlearning observation by calculating the values (real part) of the off-diagonal elements of the cross-correlation matrices $\mathbf{R}_{Y}(\omega, k)$ at each frequency bin. (a) Corresponding to the case without penalty function as in [4]. (b)-(d) Corresponding to the cases with penalty functions and penalty coefficients $\kappa$ are $1,0.1$, and 0.01 respectively.

To further evaluate the performance of the proposed method, we measure the resemblance between the original and the reconstructed source waveforms by resorting to their mean squared

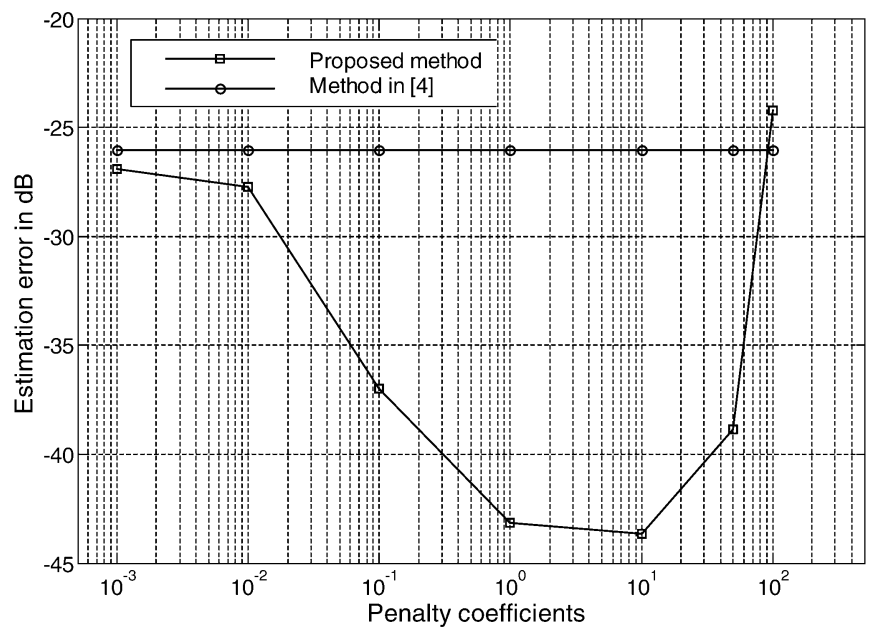

Fig. 9. Comparison of the estimate error between the proposed method and the method in [4] using waveform simularity measurement (step size $\mu=0.06$ ).

difference. By assuming the signals are zero-mean and unit-variance, we have

$$
\begin{aligned}
\varepsilon^{2}(\mathrm{~dB}) & =10 \log E\left[\|\mathbf{y}-\mathbf{s}\|^{2}\right] \\
& =10 \log \left\{\frac{1}{N} \sum_{i=1}^{N} E\left[\left(y_{i}(k)-s_{i}(k)\right)^{2}\right]\right\} .
\end{aligned}
$$

We still use the speech signals in Fig. 3 and set $\mu=0.06$ (this allows us to select a large penalty according to the previous simulations, and therefore, the convergence is smooth when $\kappa=10$ ). Other parameters are the same as those in the previous experiments. We perform seven tests by changing the penalties. The estimate error was plotted in Fig. 9 on a decibel scale, where the estimate error in [4] is not influenced by the penalties. From this simulation, we can clearly see that the separation performance is improved with an increasing penalty and can reach up to $16 \mathrm{~dB}$ when $\kappa=10$. However, with further increase of the penalties, the separation performance may degrade due to the reason discussed previously. To show the improved performance of the proposed method in reconstructing the original source signals, we give another example. The two source signals are available from [61]. Both of the signals are sampled at $22.05 \mathrm{kHz}$ with a duration of $9 \mathrm{~s}$. The samples are 16-bit 2 's complement in little endian format. One source signal is a recording of the reading sound of a man. The other is a street acoustic background. The parameters are $\kappa=0.01$ and $\mu=1$. The improved separation performance can be directly perceived through Fig. 10. The estimation errors are $\varepsilon^{2}(\mathrm{~dB})=-25.71 \mathrm{~dB}$ and $\varepsilon^{2}(\mathrm{~dB})=-38.25 \mathrm{~dB}$ for the method in [4] and the proposed method, respectively (calculated through all samples). From Fig. 10, we see that the proposed algorithm reduces the amplitude ambiguity to a lower value at the same time, and this is the direct result of the fact shown in Fig. 8.

Let us examine the separation quality by using a more complicated mixing process, which will involve more time delays 

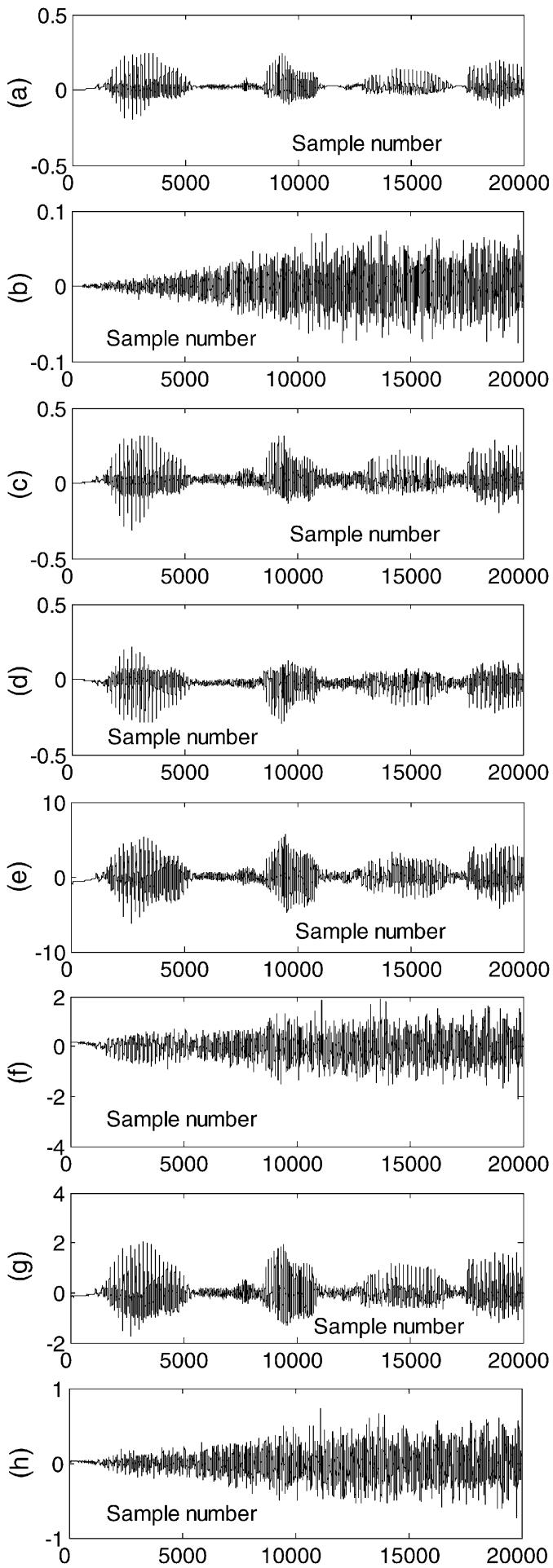

Fig. 10. Separation result of (a) a reading sound and (b) a street background sound (c), (d) mixed by a nonminimum-phase system with (g), (h) the proposed algorithm, as compared to (e), (f) the algorithm in [4].

and cross-talk between independent speech signals. The mixing matrix is

$$
\mathbf{H}(z)=\left(\begin{array}{ll}
H_{11}(z) & H_{12}(z) \\
H_{21}(z) & H_{22}(z)
\end{array}\right)
$$

where

$$
\begin{gathered}
H_{11}(z)=1+0.8 z^{-1}-0.75 z^{-2}+0.5 z^{-3}-0.4 z^{-4}- \\
0.3 z^{-5}+0.2 z^{-6}+0.15 z^{-7}-0.07 z^{-8}
\end{gathered}
$$

(a)

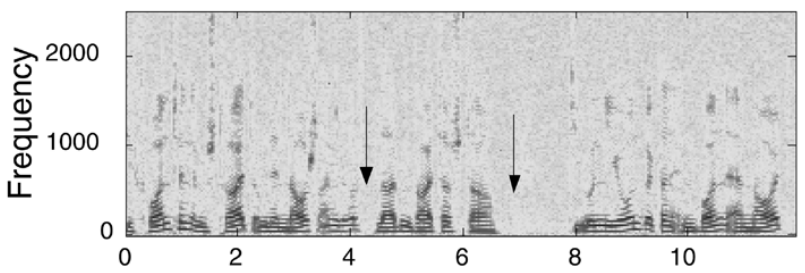

(b)

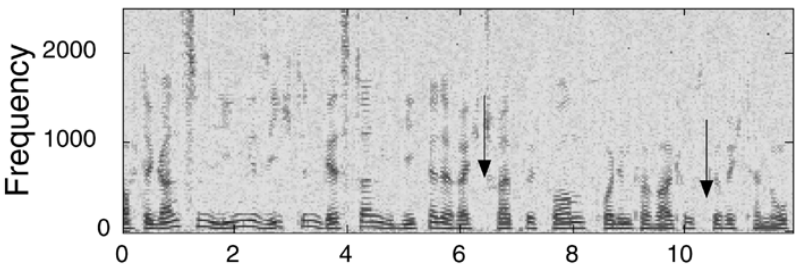

(c)

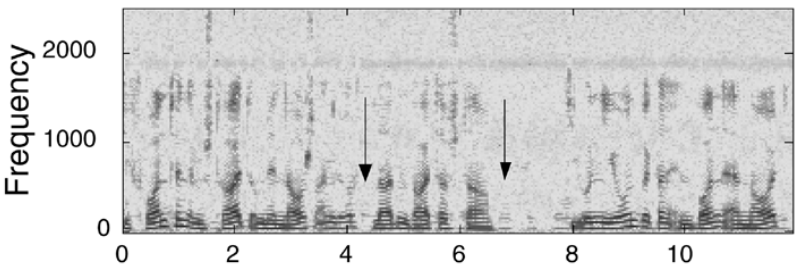

(d)

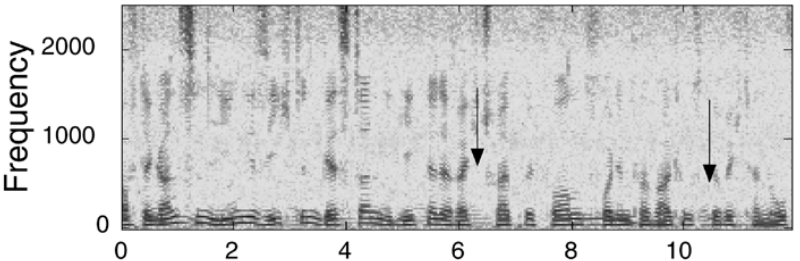

(e)

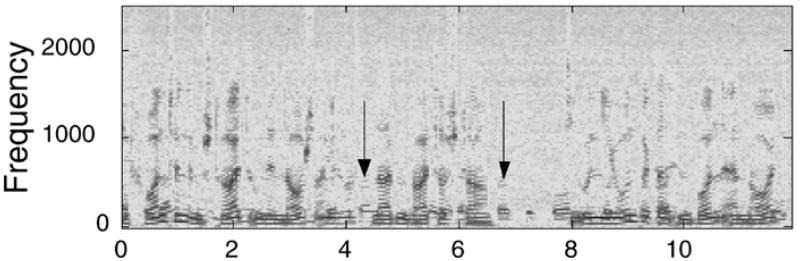

(f)

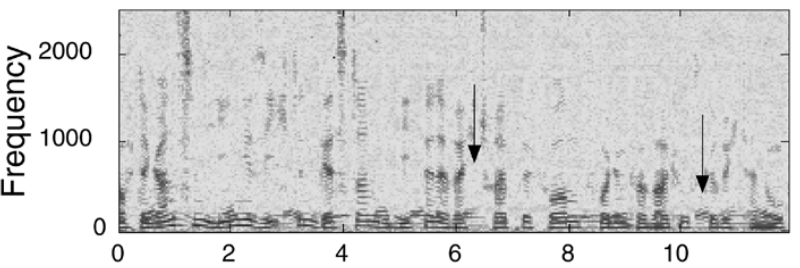

(g)
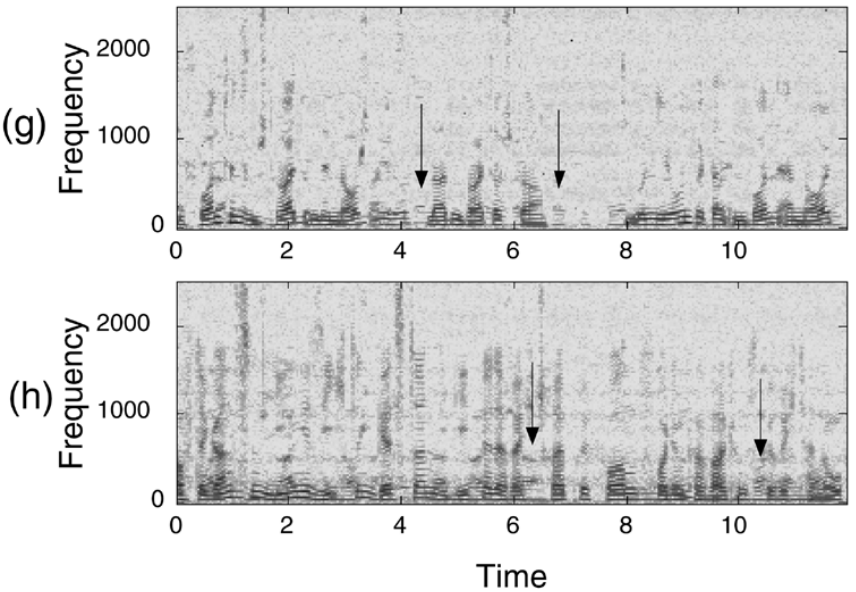

Fig. 11. Separation results of (a), (b) two speech signals mixed with FIR systems (30) with (c), (d) the proposed algorithm, as compared to the method in (e), (f) [4] and (g), (h) [62] by spectrogram. The performance comparison can be examined with the highlight arrows. 


$$
\begin{aligned}
H_{12}(z) & =0.45+0.5 z^{-1}-0.4 z^{-2}+0.4 z^{-3}- \\
& 0.3 z^{-4}-0.2 z^{-5}+0.3 z^{-6}-0.15 z^{-7}+0.1 z^{-8} \\
H_{21}(z) & =0.4-0.45 z^{-1}+0.4 z^{-2}-0.3 z^{-3}- \\
& 0.2 z^{-4}+0.3 z^{-5}-0.25 z^{-6}-0.1 z^{-7}-0.1 z^{-8} \\
H_{22}(z) & =1-0.7 z^{-1}+0.75 z^{-2}-0.5 z^{-3}+0.4 z^{-4}- \\
& 0.35 z^{-5}+0.3 z^{-6}-0.1 z^{-7}+0.08 z^{-8} .
\end{aligned}
$$

The parameters in this simulation are the same as the initial setting in the first simulation, except for $\kappa=0.08$. The step size remains the same, i.e., $\mu=0.5$, to allow comparison for the proposed method and the method in [4], which has been tuned empirically to an approximate optimum value for this test. The estimation errors of [4] and the proposed method are $\varepsilon^{2}(\mathrm{~dB})=-19.59 \mathrm{~dB}$ and $\varepsilon^{2}(\mathrm{~dB})=-28.42 \mathrm{~dB}$, respectively. The separation results are now plotted by spectrogram, which is a clearer way to examine the time-frequency representation of speech signals, and shown in Fig. 11. To calculate the spectrogram, we use a Hamming window with length 500, the size of the overlap is 450, and the length of the FFT is 512. To evaluate the separation result more generally, the comparison was made to the method in [7] as well. By close observation to Fig. 11, we found that the cross talk in the recovered signals by the proposed method has been reduced to a lower level, as compared to the other two methods.

Another method to quantify the performance is using signal to interference ratio (SIR) [4],

$$
\operatorname{SIR}[H, s]=10 \log \frac{\sum_{\omega} \sum_{i}\left|H_{i i}(\omega)\right|^{2}\left\langle\left|s_{i}(\omega)\right|^{2}\right\rangle}{\sum_{\omega} \sum_{i \neq j}\left|H_{i j}(\omega)\right|^{2}\left\langle\left|s_{j}(\omega)\right|^{2}\right\rangle} .
$$

We conduct another experiment to further evaluate the proposed method by testing the SIR improvement, which will be compared with the methods in [4] and [8], respectively. The method in [4] is a representative joint diagonalization method using the off-diagonal criterion and SOS. The approach in [8] directly implements the Jacobi angle-based joint diagonalization approach in [25] to the frequency domain BSS. Note that we use a variation of this approach so that the permutation problem is addressed in the same way as in [4]. To this end, we employed the simulated room environment, which was realized by a roommix function available from [63]. One promising characteristic of this function is that one can simulate any desired configuration of speakers and microphones. In our experiment, the room is assumed to be a $10 \times 10 \times 10 \mathrm{~m}$ cube. Wall reflections are computed up to the fifth order, and an attenuation by a factor of two is assumed at each wall bounce. We set the position matrices

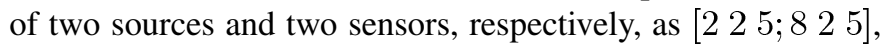
[3 $85 ; 785]$ (see Fig. 12). This setting constructs highly reverberant conditions. The parameters were set the same as those in Fig. 11. The SIR is plotted in Fig. 13, which indicates that the separation quality increases with the increasing filter length of the separation system. The performance is highly related to the data length, and it is especially clear when the filter length becomes long. Fig. 13 also indicates that incorporating a suitable penalty can increase the SIR; however, it seems that the proposed method suffers more heavily from the data length for a long filter. Additionally, incorporating a penalty may change the local minima, which can be observed from Fig. 13, as the

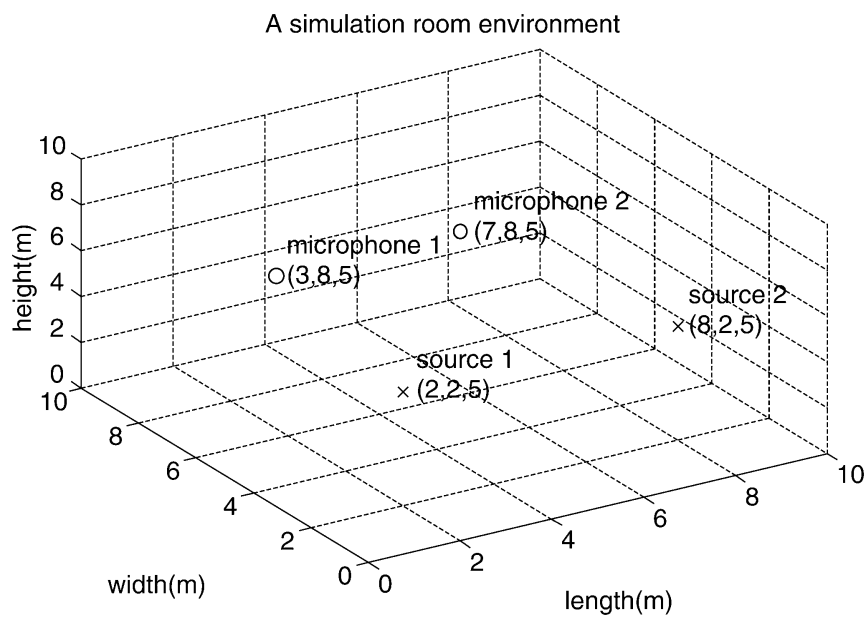

Fig. 12. Simulate a room environment using simroommix.m, where the sources are set in symmetric positions.

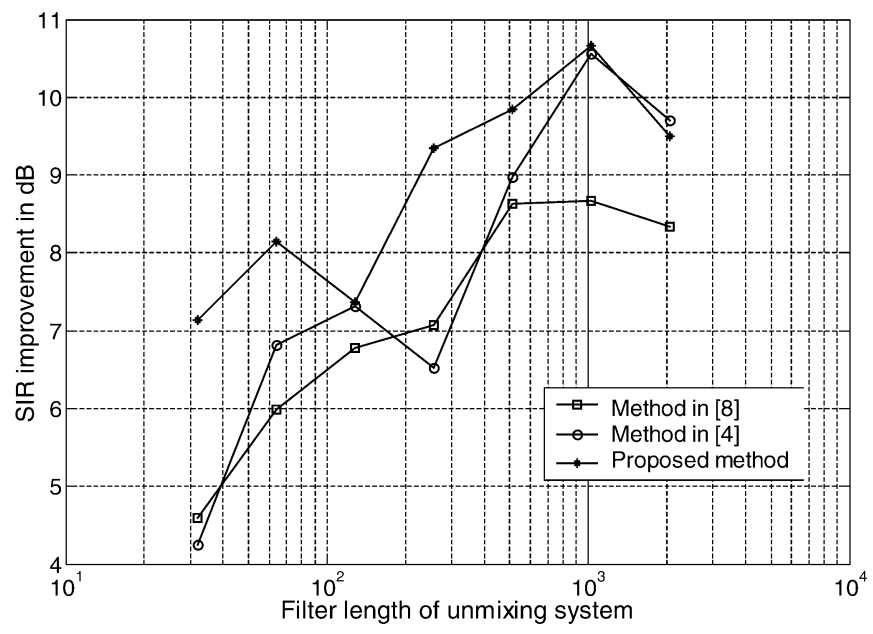

Fig. 13. SIR measurement for a simulated room environment with high reverberance.

SIR plots are not smooth, and the increased amplitude of the two methods is not consistent with each other. From Fig. 13, we can also see that exploiting spectral continuity of the separation matrix (the proposed method and that in [4]) may have superior performance to the method (e.g., [8]), which considers the separation at each frequency bin independently.

It should be noted that although the simulations are based on the nonunitary constraint, the implementation of the unitary constraint can be readily followed in a similar way. The influence (including the situation of the penalties affecting the convergence behavior and separation performance, as discussed previously in this section) of the penalty function with unitary constraint on the conventional loss criterion complies with the same regulation discussed in Sections V and VI. However, as observed in certain literature (see [22]), considering a nonunitary constraint may outperform a unitary constraint because of the unbalanced weighting of the misdiagonalization in the unitary constraint. On the other hand, it is necessary to employ a technique such as in [46] to preserve the unitary constraint during adaptation. However, a fully unitary constraint does not necessarily indicate a good separation performance (see Section VI). In practical situations, it is not possible and not 
necessary to totally fulfill the constraint. According to the simulations in this section, a finite penalty can normally generate a satisfactory solution (also see [39]).

One of the downsides of the proposed method is that although introducing a penalty function can improve the convergence performance and separation quality, it will inevitably increase the computational complexity, depending on the penalty function being used in the algorithm. Therefore, it is required to consider the practical situations of different applications when implementing the proposed method. Another problem in the implementation is that one needs to consider how much penalty should be incorporated at every iteration, and an improper penalty may lead the algorithm to fluctuation; a possible solution to this problem has been recently addressed in [60].

\section{CONCLUSIONS}

A new joint diagonalization criterion proposed for separating convolutive mixtures of nonstationary source signals in the frequency domain has been presented. Using the cross-power spectrum and nonstationarity of speech signals, the algorithm incorporates a penalty function to the conventional cost function in the frequency domain, which leads to a different search direction to find the minimum of the cost function. The new criterion transforms the separation problem into the joint diagonalization problem with unconstrained optimization, which provides a unifying way to look at the orthogonal and nonorthogonal constraint joint diagonalization methods. An implementation example with nonunitary constraint and the evaluation by numerical experiments verified the effectiveness of the proposed criterion. It has been shown that a suitable penalty function may lead the algorithm to a better performance for the separation of the convolved speech signals, in particular, in terms of shape preservation and amplitude ambiguity reduction, as compared to the second-order-based nonstationary algorithm for convolutive mixtures.

\section{APPENDIX}

DERIVATION OF (22) AND (24)

To calculate the gradient of the cost function, we can resort to the first-order Taylor expansion of the cost function with respect to a small perturbation. Suppose that the perturbation matrix of separation matrix $\mathbf{W}$ is $\Delta$. Then the perturbation of (18) reads

$$
\begin{aligned}
\mathfrak{J}(\mathbf{W}+\epsilon \boldsymbol{\Delta}) & \\
= & \mathfrak{J}(\mathbf{W})+\epsilon \mathcal{R} \mathfrak{e}\left\{\operatorname{tr}\left(\boldsymbol{\Delta}^{H} \frac{\partial \mathfrak{J}}{\partial \mathbf{W}}\right)\right\}+\mathcal{O}\left(\epsilon^{2}\right)
\end{aligned}
$$

where $\epsilon$ is a small scalar, and $\mathcal{R} \mathfrak{e}$ is the real part of a complex value. On the other hand

$$
\begin{array}{r}
\mathfrak{J}(\mathbf{W}+\epsilon \boldsymbol{\Delta})=\underset{\mathbf{W}}{\operatorname{argmin}} \sum_{\omega=1}^{T} \sum_{k=1}^{K}\{\mathcal{F}(\mathbf{W}+\epsilon \boldsymbol{\Delta})(\omega, k) \\
+\kappa \mathcal{G}(\mathbf{W}+\epsilon \boldsymbol{\Delta})(\omega, k)\}
\end{array}
$$

where $\mathcal{F}(\mathbf{W}+\epsilon \boldsymbol{\Delta})(\omega, k)$, and $\mathcal{G}(\mathbf{W}+\epsilon \boldsymbol{\Delta})(\omega, k)$ can be expanded separately

$$
\begin{aligned}
\mathcal{F}(\mathbf{W}+\epsilon \boldsymbol{\Delta})(\omega, k)= & \| \mathbf{R}_{Y}(\mathbf{W}+\epsilon \boldsymbol{\Delta})(\omega, k) \\
& -\operatorname{diag}\left[\mathbf{R}_{Y}(\mathbf{W}+\epsilon \boldsymbol{\Delta})(\omega, k)\right] \|_{F}^{2} \\
= & \left\|\mathbf{R}_{Y}(\mathbf{W}+\epsilon \boldsymbol{\Delta})(\omega, k)\right\|_{F}^{2} \\
& -\left\|\operatorname{diag}\left[\mathbf{R}_{Y}(\mathbf{W}+\epsilon \boldsymbol{\Delta})(\omega, k)\right]\right\|_{F}^{2} .
\end{aligned}
$$

Omitting the time and frequency index for simplicity and regarding (5) and (9), (34) becomes (35) and (36), shown at the bottom of the page, where $\mathbf{R}_{X}=\mathbf{R}_{X}^{H}, \mathbf{R}_{V}=\mathbf{R}_{V}^{H}$ were applied in (36).

For the unitary constraint $\mathcal{G}(\mathbf{W})(\omega, k)=\| \mathbf{W}(\omega) \mathbf{W}^{H}(\omega)-$ $\mathbf{I} \|_{F}^{2}$, we have the perturbation

$$
\begin{aligned}
\mathcal{G}(\mathbf{W} & +\epsilon \boldsymbol{\Delta})(\omega, k) \\
= & \left\|(\mathbf{W}+\epsilon \boldsymbol{\Delta})(\mathbf{W}+\epsilon \boldsymbol{\Delta})^{H}-\mathbf{I}\right\|_{F}^{2} \\
= & \left\|\left(\mathbf{W} \mathbf{W}^{H}-\mathbf{I}\right)+\epsilon\left(\mathbf{W} \Delta^{H}+\Delta \mathbf{W}^{H}\right)+\epsilon^{2} \Delta \Delta^{H}\right\|_{F}^{2} \\
= & \mathcal{G}(\mathbf{W})(\omega, k)+4 \epsilon \mathcal{R} \mathfrak{e} \\
& \times\left\{\operatorname{tr}\left(\Delta^{H} \mathbf{W}\left(\mathbf{W} \mathbf{W}^{H}-\mathbf{I}\right)\right)\right\}+\mathcal{O}\left(\epsilon^{2}\right) .
\end{aligned}
$$

For the nonunitary constraint $\mathcal{G}(\mathbf{W})(\omega, k)=\| \operatorname{diag}(\mathbf{W}(\omega)-$ I) $\|_{F}^{2}$, we have the perturbation

$$
\begin{aligned}
\mathcal{G}(\mathbf{W}+\epsilon \boldsymbol{\Delta})(\omega, k)= & \|\operatorname{diag}(\mathbf{W}+\epsilon \mathbf{\Delta}-\mathbf{I})\|_{F}^{2} \\
= & \|\operatorname{diag}(\mathbf{W}-\mathbf{I})+\epsilon \operatorname{diag}(\boldsymbol{\Delta})\|_{F}^{2} \\
= & \mathcal{G}(\mathbf{W})(\omega, k)+2 \epsilon \mathcal{R e} \\
& \times\left\{\operatorname{tr}\left(\boldsymbol{\Delta}^{H} \operatorname{diag}(\mathbf{W}-\mathbf{I})\right)\right\}+\mathcal{O}\left(\epsilon^{2}\right) .
\end{aligned}
$$

Substituting (36) and (37) into (33) and comparing with (32), we have the adaptation (22) for penalized joint diagonalization with unitary constraint. Similarly, substituting (36) and (38) into (33) and comparing with (32), we obtain the adaptation (24) for penalized joint diagonalization with nonunitary constraint. It is

$$
\begin{aligned}
\mathcal{F}(\mathbf{W}+\epsilon \boldsymbol{\Delta})(\omega, k)= & \operatorname{tr}\left\{\mathbf{R}_{Y}(\mathbf{W}+\epsilon \boldsymbol{\Delta}) \mathbf{R}_{Y}^{H}(\mathbf{W}+\epsilon \boldsymbol{\Delta})\right\}-\operatorname{tr}\left\{\mathbf{R}_{Y}(\mathbf{W}+\epsilon \boldsymbol{\Delta}) \times \operatorname{diag}\left[\mathbf{R}_{Y}^{H}(\mathbf{W}+\epsilon \boldsymbol{\Delta})\right]\right\} \\
= & \operatorname{tr}\left\{(\mathbf{W}+\epsilon \boldsymbol{\Delta})\left[\mathbf{R}_{X}-\mathbf{R}_{V}\right](\mathbf{W}+\epsilon \boldsymbol{\Delta})^{H}(\mathbf{W}+\epsilon \boldsymbol{\Delta})\left[\mathbf{R}_{X}-\mathbf{R}_{V}\right](\mathbf{W}+\epsilon \boldsymbol{\Delta})^{H}\right\} \\
& -\operatorname{tr}\left\{(\mathbf{W}+\epsilon \boldsymbol{\Delta})\left[\mathbf{R}_{X}-\mathbf{R}_{V}\right](\mathbf{W}+\epsilon \boldsymbol{\Delta})^{H} \operatorname{diag}\left((\mathbf{W}+\epsilon \boldsymbol{\Delta})\left[\mathbf{R}_{X}-\mathbf{R}_{V}\right](\mathbf{W}+\epsilon \boldsymbol{\Delta})^{H}\right)\right\} \\
= & \mathcal{F}(\mathbf{W})(\omega, k)+4 \epsilon \mathcal{R} \mathfrak{e}\left\{\operatorname{tr}\left(\boldsymbol{\Delta}^{H} \operatorname{off}\left(\mathbf{W}\left[\mathbf{R}_{X}-\mathbf{R}_{V}\right] W^{H}\right) \mathbf{W}\left[\mathbf{R}_{\mathbf{X}}-\mathbf{R}_{\mathbf{V}}\right]\right)\right\}+\mathcal{O}\left(\epsilon^{2}\right)
\end{aligned}
$$


worth noting that the adaptation (23) with respect to the noise can be obtained by following the same procedure.

\section{ACKNOWLEDGMENT}

The authors acknowledge the insightful comments provided by the Associate Editor and the anonymous reviewers, which have added much to the clarity of the paper.

\section{REFERENCES}

[1] A. S. Bregman, Auditory Scene Analysis. Cambridge, MA: MIT Press, 1990.

[2] A. Cichocki and S. Amari, Adaptive Blind Signal and Image Processing: Learning Algorithms and Applications. Chichester, U.K.: Wiley, 2002.

[3] P. Smaragdis, "Blind separation of convolved mixtures in the frequency domain," Neurocomput., vol. 22, pp. 21-34, 1998.

[4] L. Parra and C. Spence, "Convolutive blind source separation of nonstationary sources," IEEE Trans. Speech Audio Process., vol. 8, no. 3, pp. 320-327, May 2000.

[5] K. Rahbar and J. Reilly, "Blind source separation of convolved sources by joint approximate diagonalization of cross-spectral density matrices," in Proc. ICASSP, May 2001.

[6] V. Capdevielle, C. Serviere, and J. L. Lacoume, "Blind separation of wideband sources in the frequency domain," in Proc. ICASSP, 1995, pp. 2080-2083.

[7] J. Anemüller and B. Kollmeier, "Amplitude modulation decorrelation for convolutive blind source separation," in Proc. ICA, Helsinki, Finland, Jun. 2000, pp. 215-220.

[8] N. Murata, S. Ikeda, and A. Ziehe, "An approach to blind source separation based on temporal structure of speech signals," Neurocomput., vol. 41 , pp. 1-24, 2001.

[9] K. I. Diamantaras, A. P. Petropulu, and B. Chen, "Blind two-inputtwo-output FIR channel identification based on frequency domain second-order statistics," IEEE Trans. Signal Process., vol. 48, no. 2, pp. 534-542, Feb. 2000.

[10] B. Chen and A. P. Petropulu, "Frequency domain blind mimo system identification based on second- and higher-order statistics," IEEE Trans. Signal Process., vol. 49, no. 8, pp. 1677-1688, Aug. 2001.

[11] M. Z. Ikram and D. R. Morgan, "A multiresolution approach to blind separation of speech signals in a reverberant environment," in Proc. ICASSP, May 2001.

[12] S. Araki, S. Makino, R. Mukai, Y. Hinamoto, T. Nishikawa, and H. Saruwatari, "Equivalence between frequency domain blind source separation and frequency domain adaptive beamforming," in Proc. ICASSP, May 2002.

[13] M. Davies, "Audio source separation," in Mathematics in Signal Separation $V$. Oxford, U.K.: Oxford Univ. Press, 2002.

[14] W. E. Schobben and C. W. Sommen, "A frequency domain blind source separation method based on decorrelation," IEEE Trans. Signal Process., vol. 50, no. 8, pp. 1855-1865, Aug. 2002.

[15] J. Anemüller, T. J. Sejnowski, and S. Makeig, "Complex independent component analysis of frequency-domain EEG data," Neural Networks, vol. 16, pp. 1311-23, Aug. 2003.

[16] V. D. Calhoun, T. Adali, G. D. Pearlson, P. C. M. van Zijl, and J. J. Pekar, "Independent component analysis of fMRI data in the complex domain," Magn. Resonance Med., vol. 48, pp. 180-192, Jul. 2002.

[17] D. T. Pham, C. Servière, and H. Boumaraf, "Blind separation of convolutive audio mixtures using nonstationarity," in Proc. ICA, Nara, Japan, Apr. 2003, pp. 975-980.

[18] W. Wang, J. A. Chambers, and S. Sanei, "A joint diagonalization method for convolutive blind separation of nonstationary sources in the frequency domain," in Proc. ICA, Nara, Japan, Apr. 2003, pp. 939-944.

[19] S. Van-Gerven and D. Van-Compernolle, "Signal separation by symmetric adaptive decorrelation: Stability, convergence and uniqueness," IEEE Trans. Signal Process., vol. 43, no. 7, pp. 1602-1612, Jul. 1995.

[20] D. Yellin and E. Weinstein, "Multichannel signal separation: Methods and analysis," IEEE Trans. Signal Process., vol. 44, no. 1, pp. 106-118, Jan. 1996.

[21] J. H. Manton, "Optimization algorithms exploiting unitary constraints," IEEE Trans. Signal Process., vol. 50, no. 3, pp. 635-650, Mar. 2002.

[22] A. Yeredor, "Non-orthogonal joint diagonalization in the least-squares sense with application in blind source separation," IEEE Trans. Signal Process., vol. 50, no. 7, pp. 1545-1553, Jul. 2002.
[23] M. S. Bazaraa, H. D. Sherali, and C. M. Shetty, Nonlinear Programming Theory and Algorithms. New York: Wiley, 1993.

[24] E. Polak, Optimization Algorithms and Consistent Approximations. New York: Springer-Verlag, 1997.

[25] J. F. Cardoso and A. Souloumiac, "Blind beamforming for nongaussian signals," Proc. Inst. Elect. Eng. F, vol. 140, pp. 362-370, 1993.

[26] W. Wang, M. G. Jafari, S. Sanei, and J. A. Chambers, "Blind separation of convolutive mixtures of cyclostationary signals," Int. J. Adaptive Control Signal Process., vol. 18, no. 3, pp. 279-298, Apr. 2004.

[27] L. Zhang, A. Cichocki, and S. Amari, "Geometrical structures of FIR manifold and their application to multichannel blind deconvolution," in Proc. IEEE Int. Conf. Neural Network Signal Process., Madison, WI, Aug. 1999, pp. 303-314.

[28] T.-W. Lee, Independent Component Analysis: Theory and Applications. Boston, MA: Kluwer, 1998.

[29] A. J. van der Veen and A. Paulraj, "An analytical constant modulus algorithm,” IEEE Trans. Signal Process., vol. 44, no. 5, pp. 1136-1155, May 1996.

[30] A. J. van der Veen, "Joint diagonalization via subspace fitting techniques," in Proc. ICASSP, vol. 5, May 2001.

[31] D. T. Pham, "Joint approximate diagonalization of positive definite Hermitian matrices," SIAM J. Matrix Anal. Appl., vol. 22, pp. 1136-1152, 2001.

[32] G. Hori and J. H. Manton, "Critical point analysis of joint diagonalization criteria," in Proc. ICA, Apr. 2003.

[33] M. Joho and H. Mathis, "Joint diagonalization of correlation matrices by using gradient methods with application to blind signal separation," in Proc. SAM, Aug. 2002.

[34] A. Belouchrani, K. Abed-Meraim, J.-F. Cardoso, and E. Moulines, "A blind source separation technique using second order statistics," IEEE Trans. Signal Process., vol. 45, no. 2, pp. 434-444, Feb. 1997.

[35] M. Wax and J. Sheinvald, "A least-squares approach to joint diagonalization,” IEEE Signal Process. Lett., vol. 4, no. 2, pp. 52-53, Feb. 1997.

[36] S. Costa and S. Fiori, "Image compression using principal component neural networks," Image Vision Comput., vol. 19, pp. 649-668, 2001.

[37] E. Moreau, "A generalization of joint-diagonalization criteria for source separation,” IEEE Trans. Signal Process., vol. 49, no. 3, pp. 530-541, Mar. 2001.

[38] S. Fiori, "A theory for learning by weight flow on Stiefel-Grassman manifold," Neural Comput., vol. 13, no. 7, pp. 1625-1647, Jul. 2001.

[39] J. Ma and J. K. Tugnait, "A penalty function approach to code-constrained CMA for blind multiuser CDMA signal detection," in Proc. ICASSP, May 2001.

[40] M. Joho and K. Rahbar, "Joint diagonalization of correlation matrices by using newton methods with application to blind signal separation," in Proc. SAM, Aug. 2002.

[41] T. F. Coleman, J. Liu, and W. Yuan, "A quasi-Newton quadratic penalty method for minimization subject to nonlinear equality constraints," Comput. Optimization Applicat., vol. 15, no. 2, pp. 103-124, Feb. 2000.

[42] A. V. Fiacco and G. P. McCormick, Nonlinear Programming: Sequential Unconstrained Minimization Technique. New York: Wiley, 1968.

[43] A. Cichocki and R. Unbehauen, Neural Networks for Optimization and Signal Processing. New York: Wiley, 1993.

[44] L. Parra and C. Alvino, "Geometric source separation: Merging convolutive source separation with geometric beamforming," IEEE Trans. Speech Audio Process., vol. 10, no. 6, pp. 352-362, Sep. 2002.

[45] S. C. Douglas, S. Amari, and S.-Y. Kung, "On gradient adaptation with unit norm constraints," IEEE Trans. Signal Process., vol. 48, no. 6, pp. 1843-1847, Jun. 2000.

[46] S. C. Douglas, "Self-stabilized gradient algorithms for blind source separation with orthogonality constraints," IEEE Trans. Neural Networks, vol. 11, no. 6, pp. 1490-1497, Jun. 2000.

[47] T. W. Lee, A. J. Bell, and R. Lambert, "Blind separation of delayed and convolved sources," in Advances in Neural Information Processing Systems. Cambridge, MA: MIT Press, 1997, vol. 9, pp. 758-764.

[48] J.-F. Cardoso and B. Laheld, "Equivariant adaptive source separation," IEEE Trans. Signal Process., vol. 44, no. 12, pp. 3017-3030, Dec. 1996.

[49] J.-F. Cardoso, "Informax and maximum likelihood in source separation," IEEE Signal Process. Lett., vol. 4, no. 4, pp. 112-114, Apr. 1997.

[50] U. Helmke and J. B. Moore, Optimization and Dynamical Systems. New York: Springer-Verlag, 1994.

[51] A. Edelman, T. A. Arias, and S. T. Smith, "The geometry of algorithms with orthogonality constraints," SIAM J. Matrix Anal. Applicat., vol. 20, no. 2, pp. 303-353, 1998.

[52] S. Amari, T. P. Chen, and A. Cichocki, "Nonholonomic orthogonal learning algorithms for blind source separation," Neural Comput., vol. 12, pp. 1463-1484, 2000. 
[53] A. Cichocki and P. Georgiev, "Blind source separation algorithms with matrix constraints," IEICE Trans. Funda., vol. E86-A, no. 1, Jan. 2003.

[54] M. D. Plumbley, "Algorithms for nonnegative independent component analysis," IEEE Trans. Neural Networks, vol. 14, no. 2, pp. 534-543, May 2003.

[55] M. Nikpour, J. H. Manton, and G. Hori, "Algorithms on Stiefel manifold for joint diagonalization," in Proc. ICASSP, 2002, pp. 1481-1484.

[56] A. Bunse-Gerstner, R. Byers, and V. Mehrmann, "Numerical methods for simultaneous diagonalization," SIAM J. Mater. Anal. Appl., vol. 14, pp. 927-949, 1993.

[57] M. Klajman and J. A. Chambers, "Approximate joint diagonalization based on the Cayley transform," in Mathematics in Signal Separation V. Oxford, U.K.: Oxford Univ. Press, 2002.

[58] S. Ding, T. Hikichi, T. Niitsuma, M. Hamatsu, and K. Sugai, "Recursive method for blind source separation and its applications to real-time separation of acoustic signals," in Proc. ICA, Nara, Japan, Apr. 2000.

[59] M. Joho, R. H. Lambert, and H. Mathis, "Elementary cost functions for blind separation of nonstationary source signals," in Proc. ICASSP, May 2001.

[60] A. E. Smith and D. W. Coit, "Penalty functions," in Handbook of Evolutionary Computation, T. Baeck, D. Fogel, and Z. Michalewicz, Eds: A Joint Publication of Oxford University Press and Institute of Physics, 1995.

[61] P. Smaragdis.. [Online]. Available: http://sound.media.mit.edu/icabench/

[62] J. Anemüller.. [Online]. Available: http://medi.uni-oldenburg.de/members/ane

[63] A. Westner.. [Online]. Available: http://www.media.mit.edu/ westner

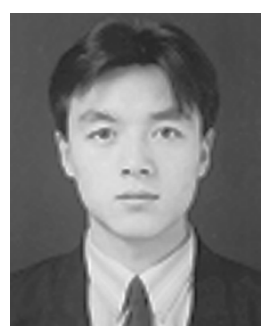

Wenwu Wang (M'03) was born in Anhui, China, in 1974. He received the B.Sc. degree in automatic control in 1997, the M.E. degree in control science and control engineering in 2000, and the Ph.D. degree in navigation guidance and control in 2002, all from Harbin Engineering University, Harbin, China.

He then joined the Department of Electronic Engineering, King's College, London, U.K., as a postdoctoral research associate and transferred to the Cardiff School of Engineering, Cardiff University, Cardiff, U.K., in January 2004. His current research interests are in the areas of blind signal processing, machine learning, and perception.

Dr. Wang is a member of the IEE and of the IEEE Signal Processing and Circuits and Systems Societies.

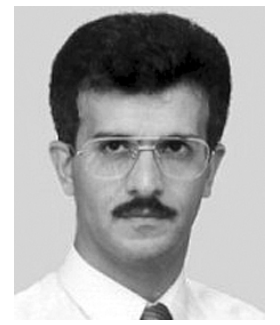

Saeid Sanei (M'97) received the B.Sc. degree in electronic engineering from Isfahan University of Technology, Tehran, Iran, in 1985 and the M.Sc. degree in satellite communication engineering from the University of Surrey, Surrey, U.K., in 1987. He received the Ph.D. degree in biomedical signal and image processing from Imperial College of Science, Technology, and Medicine, London, U.K., in 1991.

He has since held academic positions in Iran, Singapore, and the United Kingdom. He is a Senior Lecturer with the Cardiff School of Engineering, Cardiff

University, Cardiff, U.K.

Dr. Sanei is a member of the IEEE Signal Processing, Biomedical Engineering, and Communications Societies.

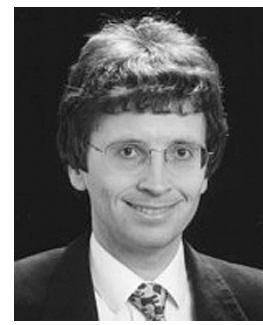

Jonathon A. Chambers (SM'98) was born in Peterborough, U.K., in 1960. He received the B.Sc. (Hons) degree from the Polytechnic of Central London, London, U.K., in 1985, together with the Robert Mitchell Medal as the top graduate of the Polytechnic, and the Ph.D. degree in 1990 after study at Peterhouse, Cambridge University, and Imperial College London.

He served in the Royal Navy as an Artificer Apprentice in Action, Data, and Control between 1979 and 1982. He has since held academic and industrial positions at Bath University, Bath, U.K.; Imperial College London; King's College London; and Schlumberger Cambridge Research, Cambridge, U.K. In January 2004, he became a Cardiff Professorial Research Fellow within the Cardiff School of Engineering, Cardiff, U.K. He leads a team of researchers involved in the analysis, design, and evaluation of novel algorithms for digital signal processing with application in acoustics, biomedicine, and wireless communications. His research contributions have been in adaptive and blind signal processing. He has authored/co-authored almost 200 conference and journal publications and supervised $20 \mathrm{Ph} . \mathrm{D}$. graduates.

Dr. Chambers has served as an Associate Editor for the IEEE TRANSACTIONS ON SIGNAL PROCESSING and Circuits and Systems. He is also a past chairman of the IEE Professional Group E5, Signal Processing. He is currently serving as an Associate Editor for the IEEE SIGNAL PROCESSING LETTERS and the IEEE TRANSACTIONS ON SIGNAL PROCESSING and as a member of the IEEE Technical Committee on Signal Processing and Methods. 